\title{
Avalanche in Adhesion at Metal Interfaces
}

Amitava Banerjea

S. N. Bose National Centre for Basic Sciences, DB-17 Salt Lake City, Calcutta 700064, India.

and

Brian S. Good

National Aeronautics and Space Administration, Lewis Research Center, Cleveland, OH 14135, U.S.A.

\section{Short Title: Avalanche at Metal Interfaces}

\begin{abstract}
Simulations have shown that as two metal surfaces approach each other, the surface layers can avalanche together when the rigid interfacial spacing falls below a critical distance. This is accompanied by a discontinuous decrease in the adhesive energy. Here we present an examination of this phenomenon for the bcc metals Fe and $W$ using the Equivalent Crystal Theory. In order to identify the circumstances under which avalanche might be inhibited, the effect of loss of registry between the two surfaces is investigated in detail. The avalanche is inhibited when the two surfaces are sufficiently far out of registry and when only a few layers near the surface are allowed to relax. As the relaxing slabs get thicker a sharp avalanche reappears. However, as the loss of registry increases the energy released in the avalanche decreases.
\end{abstract}

PACS Numbers: 68.35.-p, 46.30.Pa, 81.40.Pq 


\section{INTRODUCTION}

Recent simulation studies using different semi-empirical methods ${ }^{1-6}$ suggest that there are conditions under which solid surfaces will jump across and close an interfacial gap even when the initial interfacial separation is significantly larger than the bulk interplanar spacing. The first suggestion that solid surfaces could jump together was made by Pethica and Sutton. ${ }^{1}$ Primarily interested in the interaction of a metallic tip with a flat surface in the scanning tunneling ${ }^{7}$ and atomic force ${ }^{8}$ microscopes (STM/AFM), they drew their conclusions from studies using either a Lennard-Jones pair potential or continuum elasticity theory. Both of these approaches have serious limitations of which the authors were certainly aware. ${ }^{1}$ Subsequent investigations ${ }^{2-6}$ of the stability of adhering flat surfaces have found quantitative evidence for an avalanche effect.

Smith et al., ${ }^{2}$ studied a (100) interface between two nickel half crystals using the Equivalent Crystal Theory (ECT) ${ }^{9,10}$ which is based on the universal binding energy relation (UBER). ${ }^{11}$ Initially the atoms in each half crystal were fixed rigidly at bulk interatomic positions and the adhesive energy was computed as a function of $d_{R}$, the rigid interfacial spacing. Next, at each value of $d_{R}$ a gradually increasing number of planes parallel to the interface were allowed to relax in order to minimize the total energy. Allowing only the surface plane on each slab to relax, it was found that for large values of $d_{R}$, consistent with relaxation at free metal surfaces, ${ }^{10,12-14}$ the surface layer on either half crystal relaxed inward reducing the separation between itself and the first subsurface layer. There was a significant energy barrier preventing the surface layers from jumping across the interfacial gap. As the value 
of $d_{R}$ was decreased, the height of this energy barrier decreased until at a certain critical value of $d_{R}$ it disappeared. At that point it became energetically favorable for the two surface layers to jump across the interfacial gap and come together. In other words, when the two surfaces were pushed to within some critical distance, $d_{R}^{c r i t}$, of each other, the surface atomic layers could not be held apart. This jumping across to close the interfacial gap was rather sudden and was accompanied by a sharp, discontinuous drop in adhesive energy. The large residual elastic strain, now distributed over three interatomic spacings rather than being concentrated at the interface, was gradually relieved as the distance between the substrates was decreased farther. As a result, the post-avalanche variation of the adhesive energy with $d_{R}$ was more nearly parabolic. This was quite different from the behavior of the energy in the case of rigid adhesion which followed the UBER ${ }^{15}$ and is well described by the Rydberg function:

$$
\begin{aligned}
E(d) & =\Delta E E^{*}\left(d^{*}\right), \\
E^{*}\left(d^{*}\right) & =-\left(1+d^{*}\right) \exp \left(-d^{*}\right), \\
d^{*} & =\left(d-d_{m}\right) / l,
\end{aligned}
$$

Upon relaxing more than one atomic layers it was discovered ${ }^{2}$ that as the number of relaxed atomic layers, $n$, increased, the critical value of $d_{R}$ for avalanche to occur also increased. Smith et al. ${ }^{2}$ also argued that as $n \rightarrow \infty$, the critical distance $d_{R}^{c r i t} \sim \ln n$ and the discontinuity in the energy approaches the surface energy which is of the order of $1 \mathrm{eV}$ per surface atom.

In their first report Smith et al. ${ }^{2}$ indicated some circumstances under which avalanche at solid surfaces may be inhibited. Among other things, they suggested 
that a lack of registry across the interface may prevent, or at least severely inhibit the avalanche. In a subsequent zero-temperature Monte Carlo (MC) investigation of the avalanche phenomenon in $\mathrm{Ni}$ Good et al. ${ }^{6}$ reported that in the case of $\mathrm{Ni}$ (001) a total loss of registry - so that atoms on either side of the interface come down on top of each other - avalanche is severely inhibited in that there is no sharp drop in the energy. However, there was a significant, if rounded, avalanche-like change in the interlayer spacings. That study ${ }^{6}$ allowed no more than three surface layers on either side to relax and the authors noted that as the number of relaxing layers increased the changes in the interlayer spacings appeared to get sharper.

In this paper we present a study of avalanche in bcc metals. We have investigated avalanche at the (001) and (110) interfaces of Fe and W. Although W (001) is known to reconstruct, we have ignored reconstruction effects for the present. We also present here a more detailed study of the effect of registry on avalanche at the (110) interface of Fe. In the next section we present a brief review of the simulation procedure while in Section III we present the results for avalanche at the (001) and (110) interfaces in registry for both, $\mathrm{Fe}$ and $\mathrm{W}$ and the results for the out-of-registry (110) interfaces of Fe. Finally, we summarise the study and discuss directions of future work in Section IV.

\section{SIMULATION PROCEDURE}

In the simulations that we present here we have used the Equivalent Crystal Theory ${ }^{9,10}$ (ECT) to compute the energies of the system. This method, based on the Universal Binding Energy Relation, ${ }^{11}$ has been described in detail elsewhere. Nevertheless we present here a very brief review of the essentials. The ECT, as 
generally implemented, expresses the energy of a collection of atoms as a sum over individual atomic contributions. Each atomic contribution comprises four different terms. The first of these terms depends essentially on the local density in the immediate neighborhood of the atom in question and is generally the largest single contribution to the surface or interface energy. The second term accounts for local deviations in symmetry away from that of the ground-state crystal and local variations in nearest-neighbor distances. The other two terms depend on changes in bond angles and account for shear-like distortions. The ECT has been shown to give accurate surface energies and surface relaxations for a variety of materials. ${ }^{8,10,12,13}$ In particular, it has been shown ${ }^{13}$ that the last two, bondangle-dependent terms contribute little to the relaxation energies of metal surfaces. Hence, in this study we have neglected the last two terms of the ECT energy expression.

This study has been restricted to planar relaxations only, i. e., each atomic layer parallel to the interface is assumed to retain its planar structure as the system relaxes - no in-plane reconstruction or buckling of atomic planes is permitted. In order to relax the system the energy was minimized with respect to the various interlayer spacings using a Monte Carlo procedure. The energy of the system two semi-infinite solids with parallel surfaces either in or out of registry - was calculated at each value of the rigid interfacial separation, $d_{R}$, as a function of the interplanar spacings in either half solid. The variations in interlayer spacings were assumed to be symmetric about the interface. That is, the distance between layers $i$ and $i+1, d_{i, i+1}$, had the same value in the two half solids. Hence, when allowing $n$ layers to relax one needed to be concerned with only $n$ independent variables 
and to compute the energy of $n+2$ layers in only one of the semi-infinite slabs. As a result the energies presented here are half of the total system energies of adhesion and the depth of the energy well is the surface energy of the appropriate metal surface.

It should be noted that these calculations do not make use of the complete Metropolis Monte Carlo algorithm ${ }^{16}$ in that the temperature of the system is assumed to be zero and there is no provision made here for escape from a local energy minimum. The energy surfaces are rather smooth and the general locations of the energy minima are known. As discussed earlier, for a given $d_{R}$ there is either one minimum (for those values of $d_{R}$ for which avalanche does occur) or two minima (for those values of $d_{R}$ where avalanche does not occur). One of the minima occurs when all relaxing layers are closer to each other than to their repsective bulk slabs. In the non-avalanche case, there is a second (local) minimum which occurs when all relaxing layers are close to their respective bulk slabs. Avalanche occurs when the energy barrier between this minimum and the global one with the relaxing layers closer to each other disappears and the local minimum gives way to an inflection point. It is precisely the transition from a two-minimum configuration to a single-minimum one (as $d_{R}$ is decreased) and the associated transition of the system from a local energy minimum to a global energy minimum that we wish to investigate. This is why we have not implemented the full Metropolis MC algorithm. However, we do plan to implement the full Metropolis MC scheme soon - allowing individual atoms their freedom in a larger computational cell instead of requiring a planar movement - in order to investigate the effect of temperature on the phenomenon of avalanche. 


\section{RESULTS}

\section{A. Interfaces in registry}

The variation of relaxed binding energy with initial rigid interfacial spacing, $d_{R}$, for the case with the approaching surfaces in perfect registry, is shown in Figs. 1 and 2 for the (001) and (110) interfaces of Fe and W. The sudden drop in the energy upon avalanche is clearly evident.

The physical transformation associated with this precipitous drop in energy is seen clearly in Figs. 3-5. Figure 3 shows - for different interfaces (in perfect registry) and different numbers of relaxing layers as indicated - plots of the final separation between the relaxed surface layers on either side of the interface, also measured with respect to the equilibrium interplanar spacing, as a function of $d_{R}$. At large values of $d_{R}$, when the surfaces are essentially isolated and not interacting, the separation between surface layers is actually slightly greater than $d_{R}$. This implies that the surface layer relaxes in toward the bulk - a phenomenon known from experiment as well as theory. ${ }^{10,12-14}$ As $d_{R}$ decreases, this separation decreases slightly, indicating a gradual reduction in the inward relaxation of the surface layer and indeed even a slight outward relaxation as the interaction across the interface increases in strength. At a certain "critical" value of $d_{R}$, however, there is a precipitous drop in the separation between surface layers, as they leave their respective slabs and come together in the center.

In Figs. 4 and 5 we have plotted the values of the different interplanar separations, $d_{i, i+1}$ against $d_{R}$ for some of the cases shown in Fig. 3. Here we see essentially a repeat of the phenomenon seen in Fig. 3. Although the effect is hard 
to detect on the scale of these figures, for large values of $d_{R}, d_{i, i+1}$ is greater or smaller than the equilibrium (bulk) value depending on whether $i$ is even or odd. This is a reflection of the well-known damped oscillatory nature of surface relaxation. ${ }^{10,12-14}$ Again, at a critical value of $d_{R}$ there is a dramatic change, this time an increase in $d_{i, i+1}$ indicating that the relaxed layers have separated from their respective slabs and form an elastically strained zone between the two bulk slabs. When more than one layer is relaxed, it is curious that while the changes in almost all the $d_{i, i+1}$ are sharp increases followed by an almost linear decrease, the behavior of $d_{1,2}$ and $d_{n, n+1}$ show a slower rounded increase after the initial steep rise before they too settle into a near-linear decrease to the equilibrium value.

As one of the objectives of this study was to investigate the behavior of $d_{R}^{\text {crit }}$, the critical value of $d_{R}$ for avalanche to occur, with the thickness of the relaxing slab, we have plotted in Fig. 6 the variation of $d_{R}^{\text {Git }}$ with $n$, the number of relaxing layers. As the exact point of avalanche in our simulations is somewhat dependent on the history of the simulation, the values of $d_{R}^{\text {crit }}$ are impossible to determine precisely. Hence, we have attempted to estimate an uncertainty in the values of $d_{R}^{c r i t}$ which is reflected in the error bars on the points. Anticipating a logarithmic variation of $d_{R}^{c r i t}$ with $n$, as discussed in the Introduction, we have also plotted a least squares fit to a logarithmic function to the data. The actual values of the parameters and the regression are indicated on the graphs. It might be argued that the quality of fit is not excellent. We believe this is because the basis for expecting a logarithmic variation - arguments involving continuum elasticity theory and the primarily exponential tail of the force $v s . d_{R}$ curve in the case of rigid adhesion - is sorely tested in avalanche involving only a handful of atomic layers near the 
interface. As the thickness of the relaxing slab increases, one would expect to find a better fit to a logarithmic function.

\section{B. Interfaces out of registry}

So far we have presented results for avalanche at two different interfaces, (001) and (110), of two different metals, Fe and W, when the two approaching surfaces are in perfect registry with each other so that when the value of $d_{R}$ is reduced to zero the interface disappears and the two halves join to make a complete, infinite, solid. We have seen that the results are very similar for the two metals and for the two interfaces. In this section we present results for cases when the two approaching surfaces are not in registry, i. e., starting from the case of perfect registry, one half solid has been shifted relative to the other by small amounts in a direction parallel to the interface.

In a previous study ${ }^{6}$ we had presented preliminary results for avalanche in the case of out-of-registry approach for the (001) interface of Ni where the shift had been by half the distance between neighboring surface atoms so that atoms on either surface layer came down on top of one another as $d_{R}$ was reduced to zero. In that paper we had also allowed up to three layers to relax. We had reported that the "total" loss of registry strongly inhibited avalanche. However, we had pointed out indications that the transitions, which were rounded rather than sharp for total loss of registry, grew sharper as the number, $n$, of relaxing layers increased. This raised the possibility that if $n$ increased to macroscopic numbers avalanche would no longer be inhibited. In this section we investigate this phenomenon for the $\mathrm{Fe}(110)$ interface. We do not present results for out-of- 
registry $W(110)$ separately because, as we have already seen for the in-registry case in the previous section, they are essentially similar to those for iron.

Figures 7 and 8 show plots of relaxed interfacial spacing and relaxed adhesive energy, respectively, against $d_{R}$ for out-of-registry $\mathrm{Fe}(110)$ interfaces with one and five layers relaxing. Results are shown for different amounts of shift, as labeled, in the (110) direction. We can see from Fig. 8 that for the cases of shifts intermediate between perfect registry and total loss of registry there appears to be no sharp drop in energy associated with avalanche when only one surface layer is allowed to relax. However, upon allowing more layers to relax we do see a sharp discontinuity in the energy associated with avalanche. Figure 7 shows the associated change in the relaxed interfacial separation. There is a sharp discontinuity in the rate of change of the interfacial separation with $d_{R}$ associated with the discontinuity in adhesive energy. This is made clearer in Figs. 9 and 10 where we have plotted the values of the interlayer spacings as functions of $d_{R}$ for the situations depicted in Figs. 7 and 8 . Figure 9 shows results for one layer relaxing while Fig. 10 shows results for the case of 5 layers relaxing. We see that even with only one layer relaxing there is a sharp change in the behavior of the interlayer spacing at a critical value of $d_{R}$ although the subsequent variation of $d_{1,2}$ is quite different for the out-of-registry cases from that seen in the case of perfect registry. This indicates that there is avalanche of sorts occuring even with one layer relaxing. From the small size of the drop in energy seen with five relaxing layers, we conclude that there might be an energy drop even in the one-layer case but that it is too small to be observed in our computations. 


\section{DISCUSSION AND SUMMARY}

The phenomenon of avalanche can be viewed as a competition between the attractive interaction of a surface layer with the layer(s) directly below in the same slab, and with the surface layer(s) across the interface in the other slab. At large values of $d_{R}$, the surface layer only interacts with other layers in the same slab, and thus it moves in toward the bulk of the slab. As $d_{R}$ decreases, however, the surface layer begins to experience an attractive interaction with the layers on the other side of the interface. Eventually there comes a point at which forming bonds across the interface lowers the energy sufficiently to compensate for the increase in energy caused by stretching the bonds between the surface layer and its neighboring bulk layer. At this point avalanche occurs.

The suppression of the effect in the totally out-of-registry case for thinner slabs, at least, can be understood by looking at the surface geometries. In the in-registry case, each (110) surface atom is bonded to two atoms in the neighboring bulk layer, four atoms in the same layer, and (for small enough $d_{R}$ ) two atoms across the interface. Thus the surface atom will begin to interact with the two atoms across the interface at the same time, and the interactions will be equal in magnitude as long as perfect registry is maintained. On the other hand, upon total loss of registry, a surface atom is bonded to two atoms in the neighboring bulk layer, and four in the same layer, all within the same slab. In the other slab, however, there is only one "nearest-neighbor" atom directly across the interface. As the slabs approach each other, the interaction across the interface is substantially weaker (relative to interactions with atoms in the same 
slab) than in the in-registry case. There are two other atoms in the surface plane across the interface which might be called "secondary" near neighbors rather than second-nearest neighbors. The interactions with these, however, will be strongly screened, especially at small values of $d_{R}$. Consequently, these cannot compensate for the decreased coordination across the interface. This is what inhibits the sharp avalanche in the out-of-registry case.

As the number, $n$, of relaxing layers increases, however, the deeper layers play a significant role and a significant amount of energy can be gained by relaxing these outward. Now, when the surface layer moves out in order to "gain" coordination the increase in energy caused by stretching the bonds with the substrate can be minimized by distributing the "strain" over several interlayer bonds. This is what causes a return of avalanche when more layers are allowed to relax. This leads us to believe that for experimentally relevant thicknesses loss of registry will not totally suppress avalanche. However, as is evident from a comparison of Figs. 1 and 2 with Fig. 7 or Figs. 4 and 5 with Figs. 8 and 9, the value of $d_{R}^{c r i t}$ does decrease as we move farther out of registry. Hence, loss of registry does appear to inhibit avalanche without suppressing it totally.

In summary, we have carried out a Monte Carlo investigation of avalanche in Fe and $\mathrm{W}$. In agreement with previous work, ${ }^{1-6}$ we find that an adhesive avalanche effect may be observed when two metal surfaces are brought into proximity. When the interfacial gap between these surfaces falls below a critical value (which depends on the number of layers relaxed), the relaxing surface layers will move away from their respective bulk substrates and form a strained zone between them. 
We have extended these previous results by considering in detail the effects of registry on the avalanche phenomenon. We find that the avalanche transition is sharp when the two surfaces are in exact registry. When the surfaces are out of registry with each other the transition is no longer as pronounced but that avalanche occurs nonetheless if several layers on either side are allowed to relax. We further find that the critical distance for avalanche, $d_{R}^{c r i t}$, and the energy released upon avalanche both decrease as the loss of registry increases.

It must be pointed out here that no lateral movement parallel to the interface was permitted in these simulations. It is possible that when several layers are allowed to relax both perpendicular and parallel to the interface they may move laterally in order to restore registry and thus facilitate the avalanche process. This would introduce an additional shear-type strain in the relaxing zone but this increase in energy may be more than compensated for by the energy released upon avalanche. This is an aspect of avalanche into which we are currently looking. We are also currently in the process of incorporating non-zero temperatures into atomistic simulations on larger unit cells as we have already mentioned in the Introduction.

\section{ACKNOWLEDGEMENTS}

The authors would like to acknowledge many useful and stimulating discussions with Drs. John Ferrante and John R. Smith. AB would like to thank the NASA Lewis Research Center for their hospitality during the course of part of this work. 


\section{References}

1. J. B. Pethica and A. P. Sutton, J. Vac. Sci. Technol. A 6, 2494 (1988).

2. John R. Smith, Guillermo H. Bozzolo, Amitava Banerjea, and John Ferrante, Phys. Rev. Lett. 63, 1269 (1989).

3. P. A. Taylor, Jeffrey S. Nelson, and B. W. Dodson, Phys. Rev. B 44, 5834 (1991).

4. R. M. Lynden-Bell, Surf. Sci. 244, 266 (1991).

5. Jeffrey S. Nelson, B. W. Dodson, and P. A. Taylor, Phys. Rev. B 45, 4439 (1992).

6. Brian S. Good, A. Banerjea, Guillermo H. Bozzolo, and John Ferrante, Mat. Res. Soc. Symp. Proc. 193, 313 (1990).

7. G. Binnig, H. Rohrer, Ch. Gerber, and E. Weibel, Phys. Rev. Lett. 49, 57 (1982).

8. G. Binnig, C. F. Quate, and Ch. Gerber, Phys. Rev. Lett. 56, 930 (1986).

9. John R. Smith and Amitava Banerjea, Phys. Rev. Lett. 59, 2451 (1987).

10. John R. Smith and Amitava Banerjea, Phys. Rev. B 37, 10411 (1988); J. R. Smith, Tom Perry, Amitava Banerjea, J. Ferrante, and G. H. Bozzolo, Phys. Rev. B 44, 6444 (1991).

11. Amitava Banerjea and John R. Smith, Phys. Rev. B 37, 6632 (1988), and references therein.

12. John R. Smith and Amitava Banerjea, J. Vac. Sci. Technol. A 6, 812 (1988). 
13. A. Rodriguez, G. H. Bozzolo, and John Ferrante, Surface Science 289, 100 (1993); ibid., 307/309, 625(1994); G. H. Bozzolo, A. Rodriguez, and John Ferrante, Surf. Sci. 315, 204 (1994).

14. F. Jona, and P. M. Marcus, in The Structure of Surfaces II, ed. by J. F. van der Veen and M. H. van Hove (Springer, 1988) pp. 90-99.

15. Amitava Banerjea, John Ferrante, and John R. Smith, Mat. Res. Soc. Symp. Proc. 140, 89 (1989); Amitava Banerjea, John R. Smith, and John Ferrante, J. Phys.: Cond. Matter Lett. 2, 8841 (1990).

16. N. Metropolis, A. W. Rosenbluth, M. N. Rosenbluth, A. H. Teller, and E. Teller, J. Chem. Phys. 6, 1087 (1953). 


\section{Figure Captions}

Figure 1. Plots of relaxed energy versus rigid interfacial separation, $d_{R}$, for Fe (a) (001), (b) (110) interfaces.

Figure 2. Plots of relaxed energy versus rigid interfacial separation, $d_{R}$, for $\mathrm{W}$ (a) (001), (b) (110) interfaces.

Figure 3. Plots of relaxed interfacial separation versus $d_{R}$ for (a) $\mathrm{Fe}(001)$, (b) $\mathrm{Fe}(110),(\mathrm{c}) \mathrm{W}(001)$, and (d) W(110) interfaces.

Figure 4. Plots of interplanar spacings, $d_{i, i+1}$ for Fe: (a) (001) interface with 1 layer relaxing, (b) (001) interface with 5 layers elaxing, (c) (001) interface with 7 layers relaxing, (d) (110) interface with 5 layers relaxing.

Figure 5. Plots of interplanar spacings, $d_{i, i+1}$, for W as in Fig. 4.

Figure 6. Values of $d_{R}^{\text {crit }}$ vs. $\mathrm{n}$, the number of relaxing layers, plotted for (a) $\mathrm{Fe}(001)$ and $\mathrm{Fe}(110)$, and (b) $\mathrm{W}(001)$ and $\mathrm{W}(110)$ as labeled. The solid lines show least-squares fits to a logarithmic function. The fitted functions and the vaves of the correlation are shown on each plot.

Figure 7. Plots of relaxed interfacial separation versus $d_{R}$ for $F e(110)$ interfaces out of registry. One surface has been shifted relative to the other in the (hkl) direction by varying amounts - in units of $d_{h k l}$, the equilibrium interplanar distance - as indicated on each of the graphs. Results shown are for cases with 1 and 5 surface layers relaxing.

Figure 8. Relaxed energy vs. $d_{R}$ plotted for $\mathrm{Fe}(110)$ interfaces out of registry. One surface has been shifted relative to the other by varying amounts 
- in units of $d_{h k l}$, the equilibrium interplanar distance - as indicated by the vector $\mathbf{b}$ on each of the graphs. In each graph results are shown for cases with 1 and 5 layers relaxing as indicated in the legend.

Figure 9. Plots of interplanar spacings, $d_{i, i+1}$ for $\mathrm{Fe}(110)$ interfaces out of registry. One surface has been shifted relative to the other in the (hkl) direction by varying amounts - in units of lattice constant - as indicated on each of the graphs. Results shown are for cases with 1 surface layer relaxing.

Figure 10. Plots of interplanar spacings, $d_{i, i+1}$ for $\mathrm{Fe}(110)$ interfaces out of registry. One surface has been shifted relative to the other in the (hkl) direction by varying amounts - in units of $d_{h k l}$, the equilibrium interplanar distance - as indicated on each of the graphs. Results shown are for cases with 5 surface layers relaxing. 


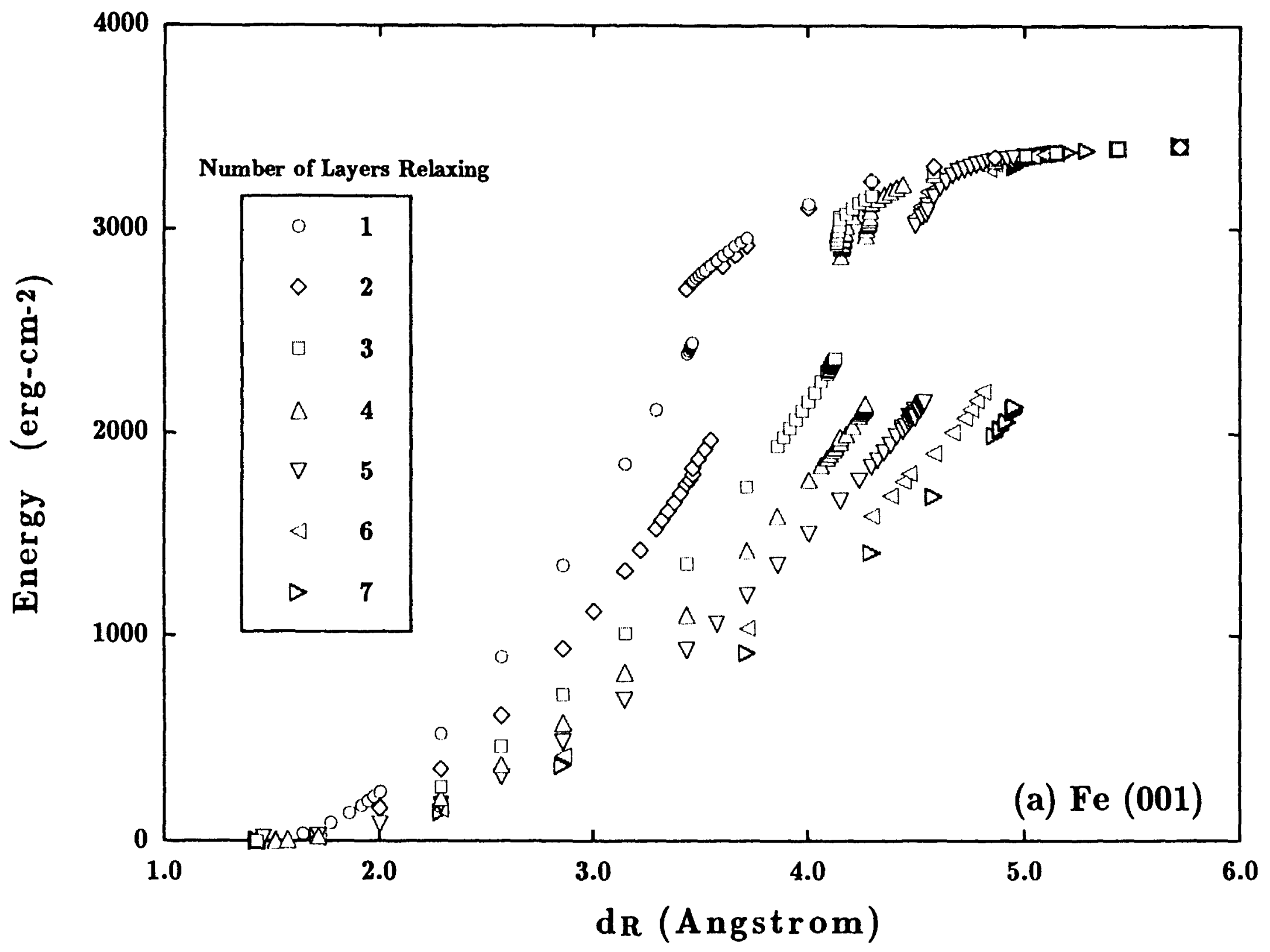




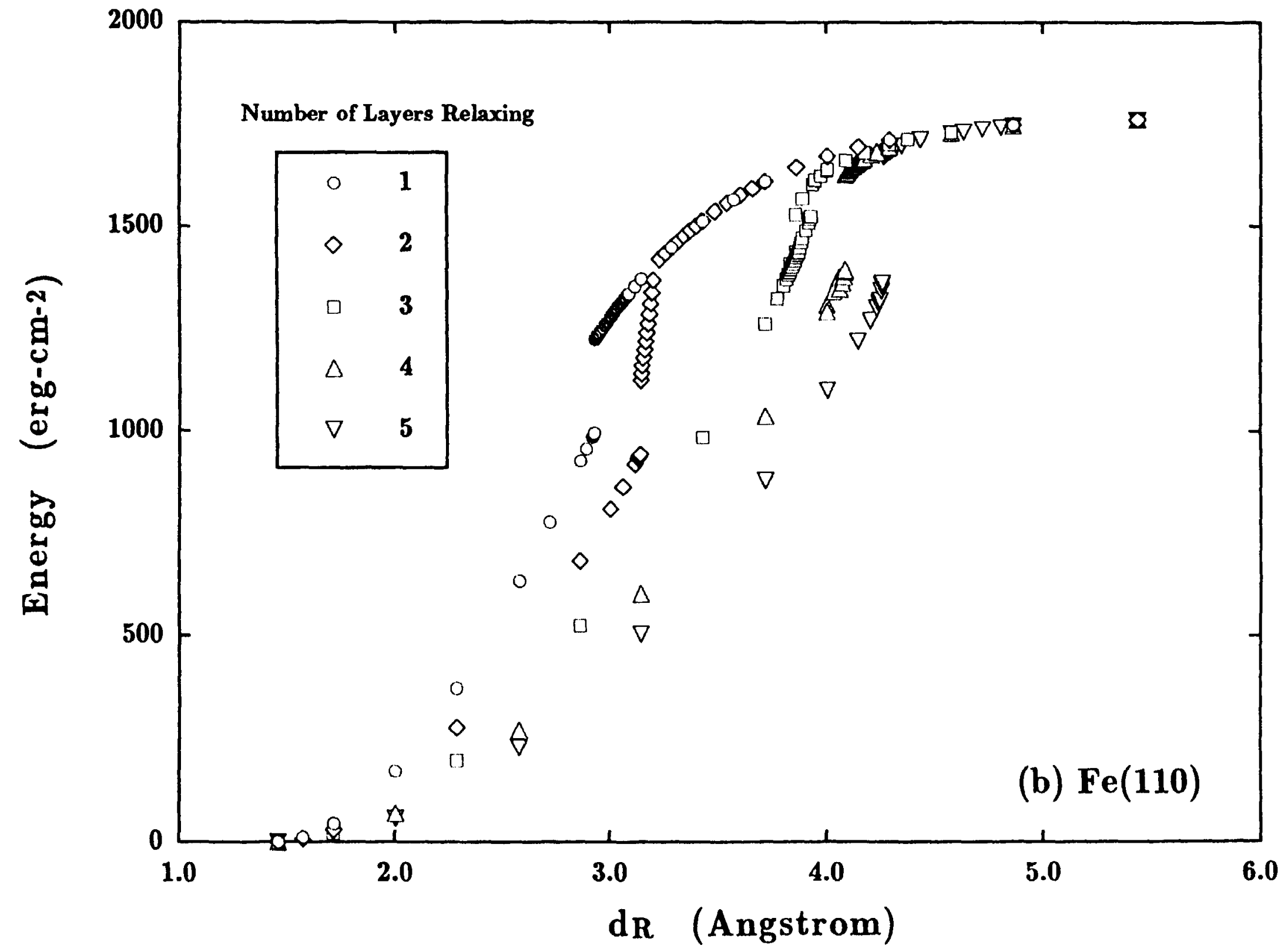




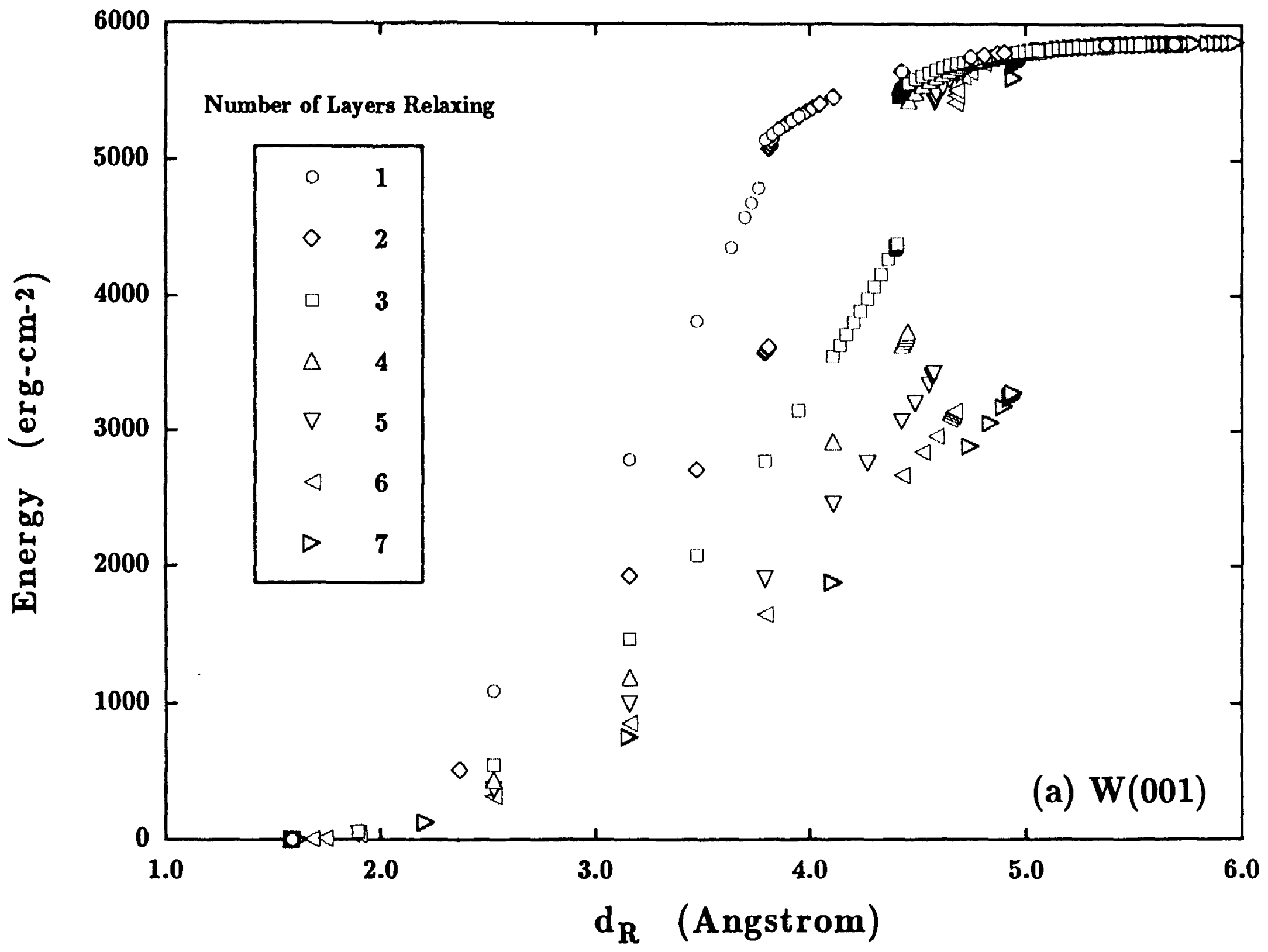




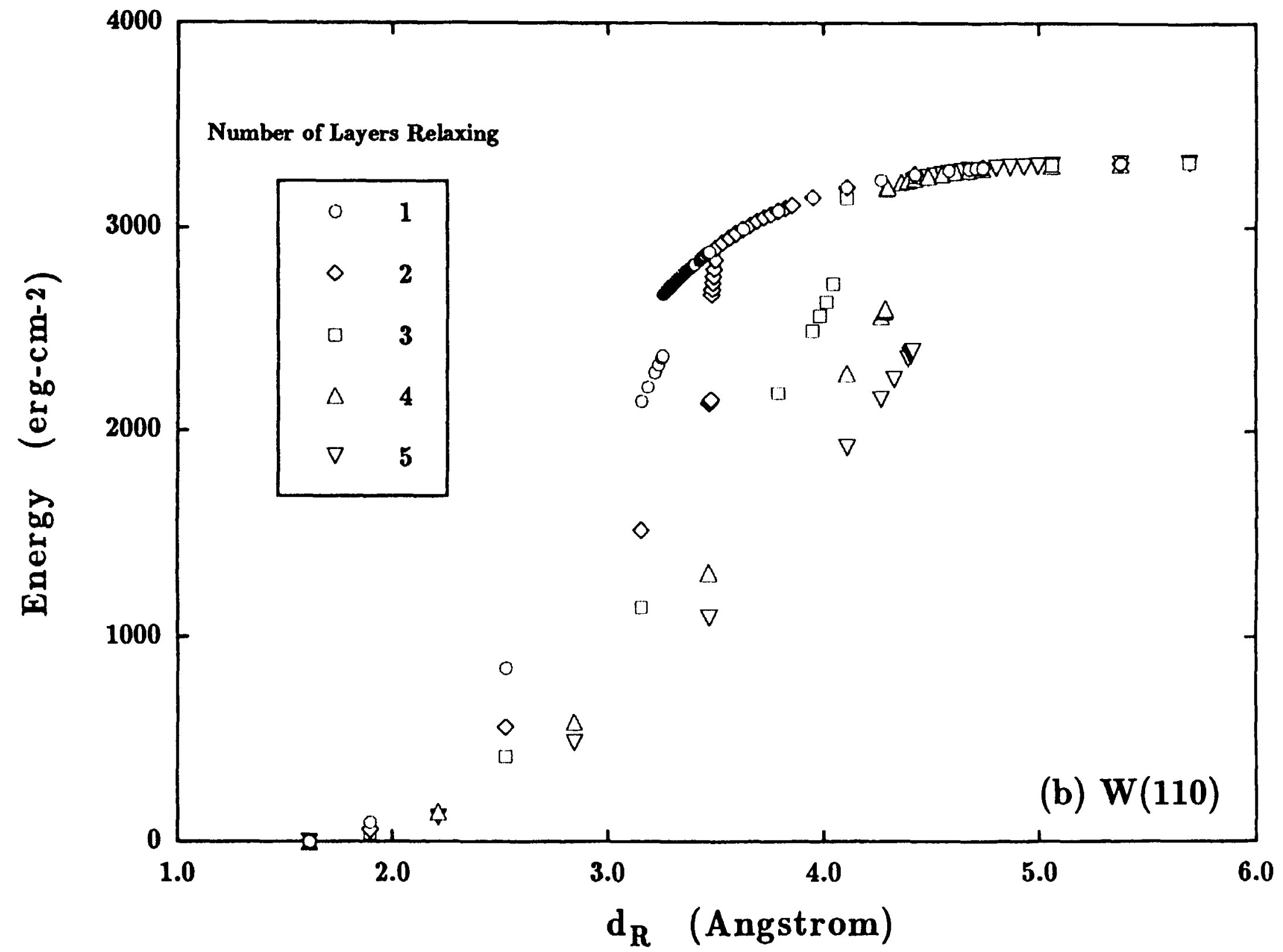




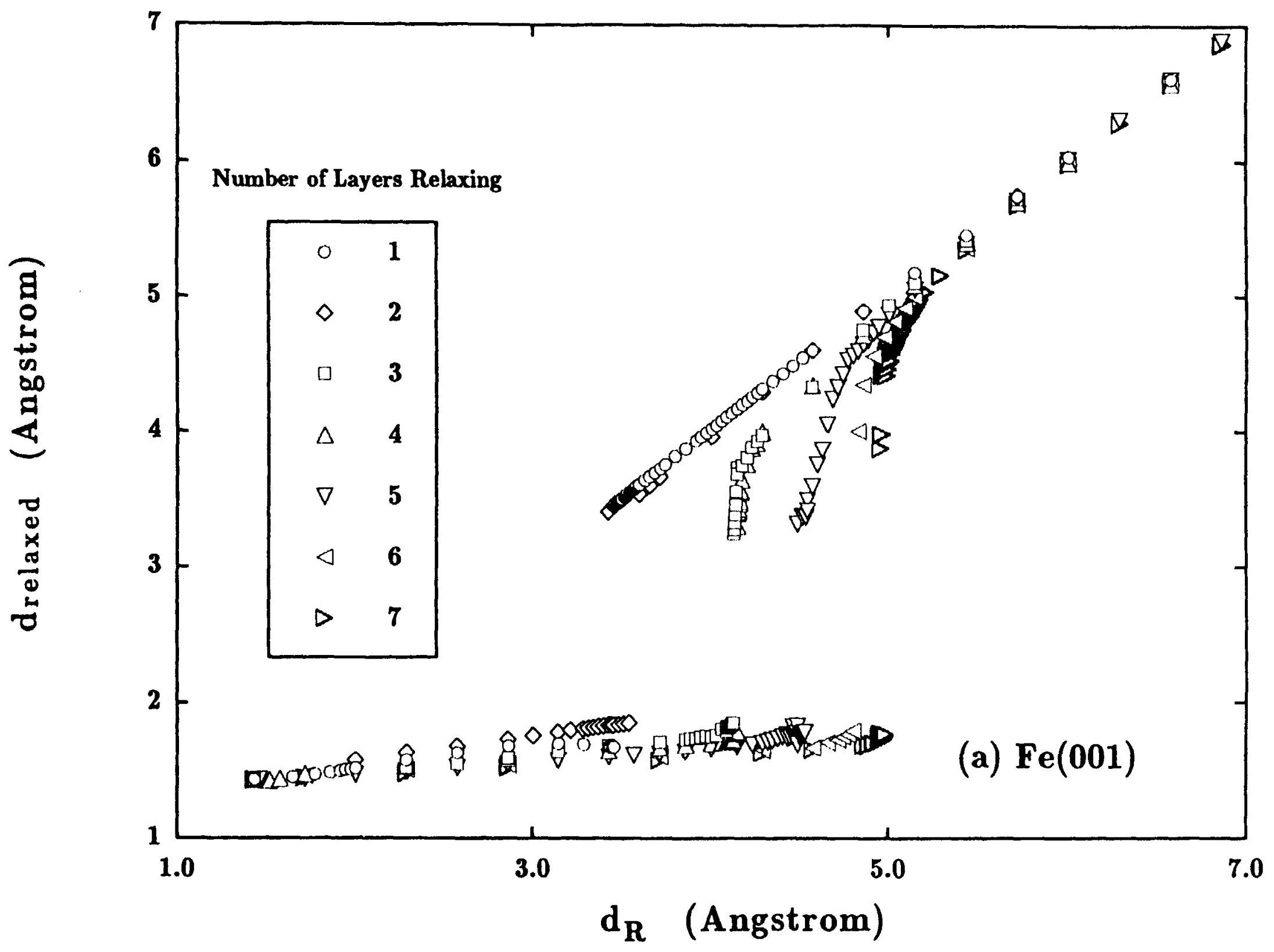




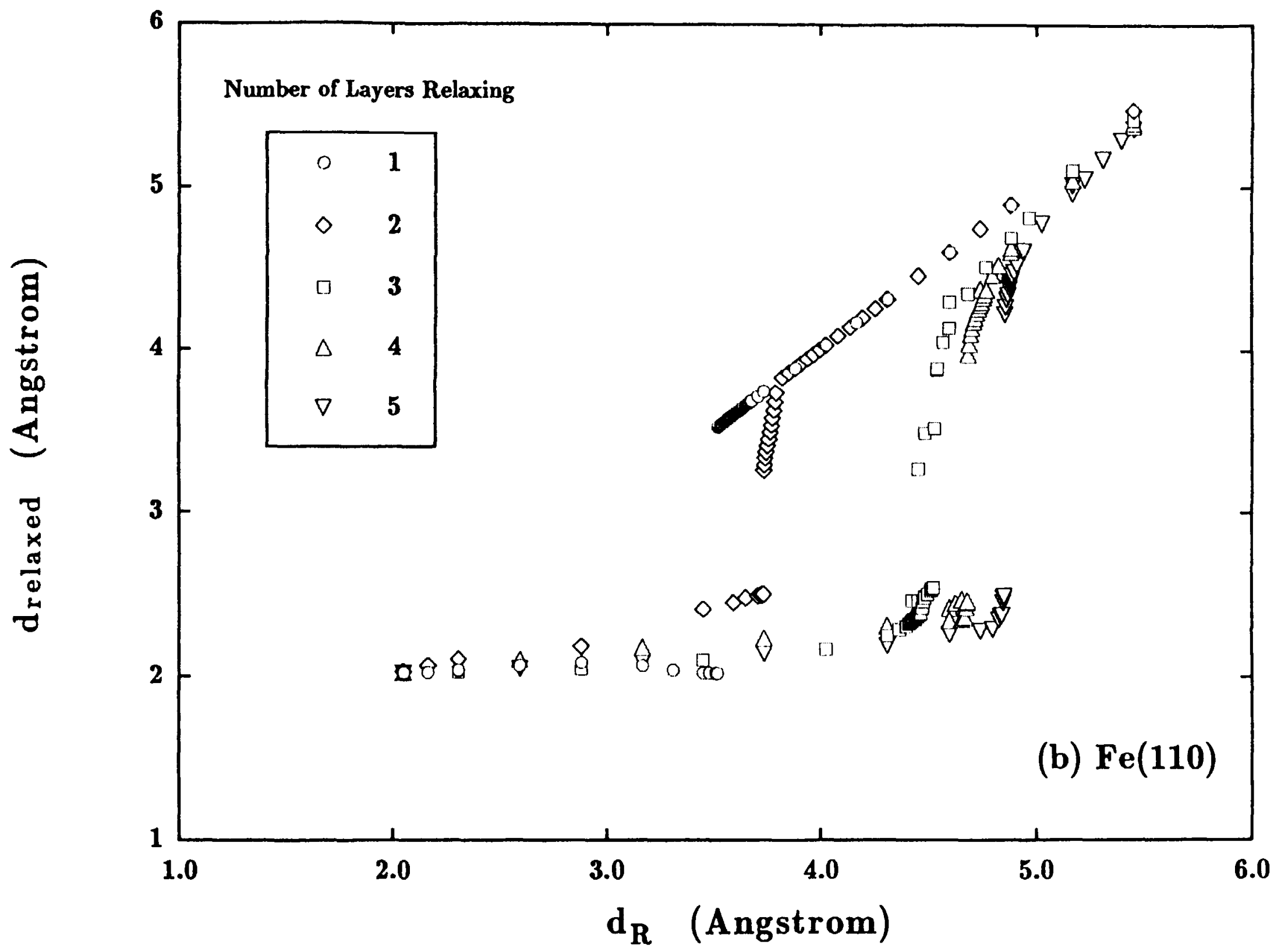




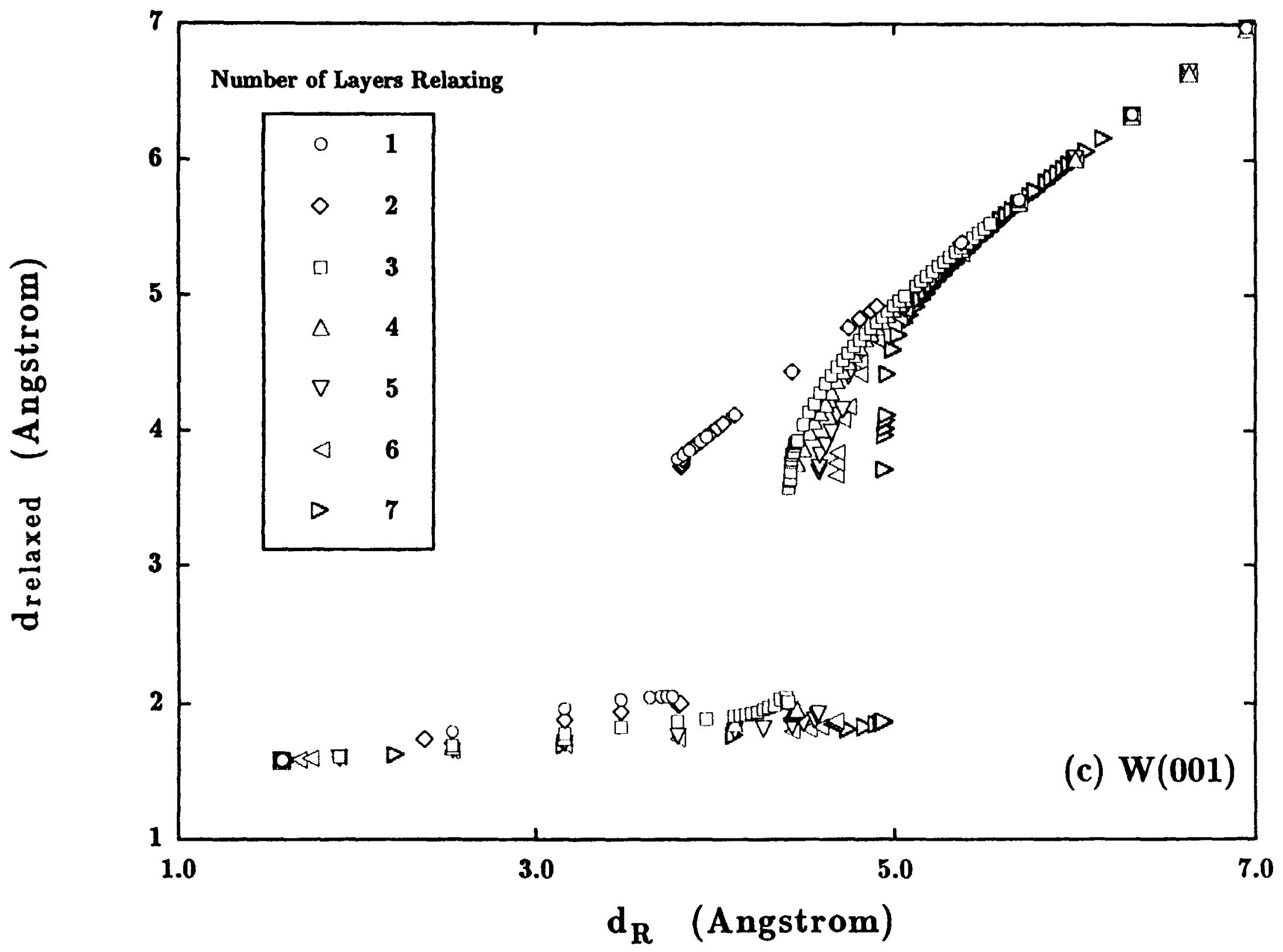




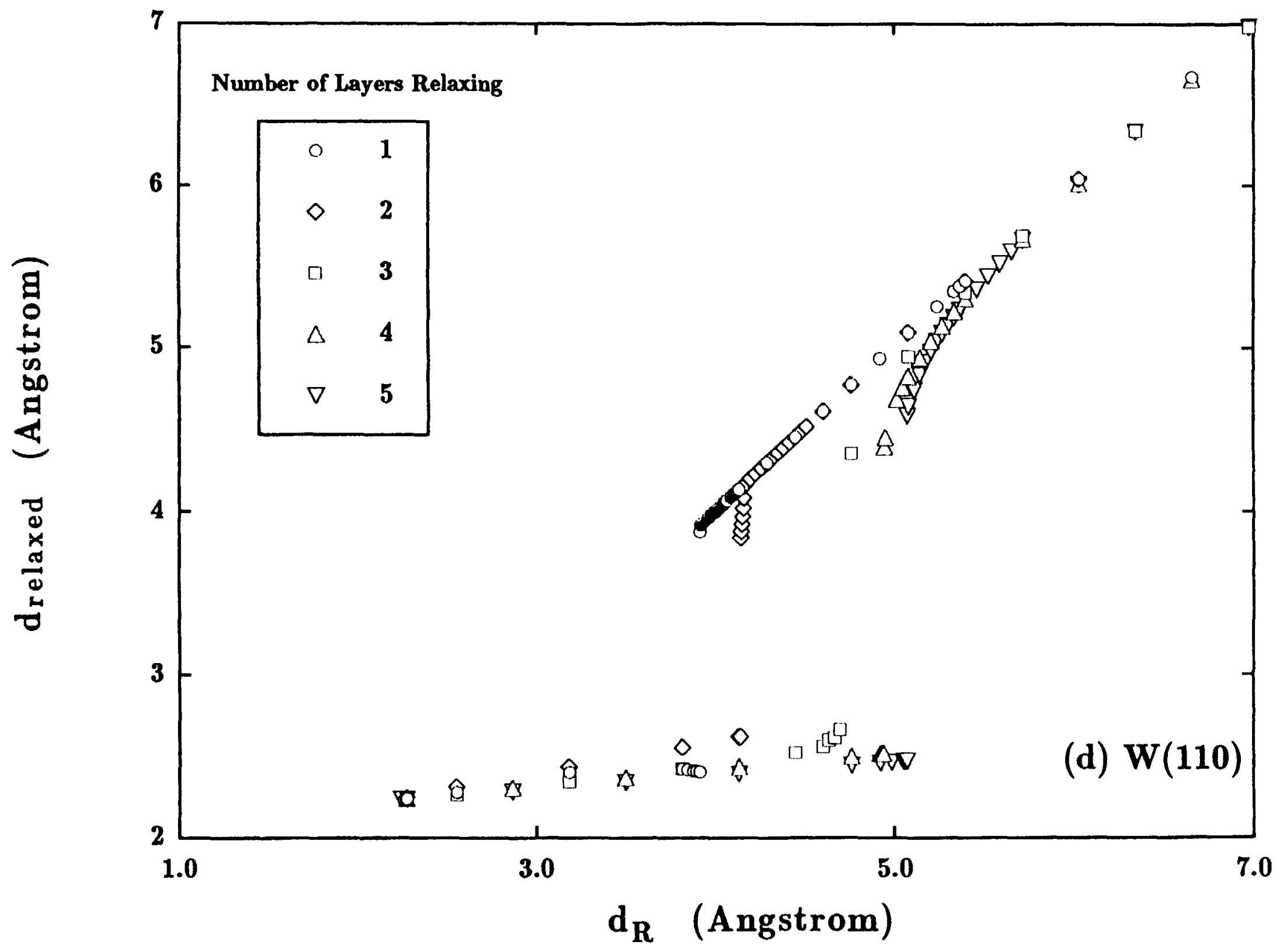




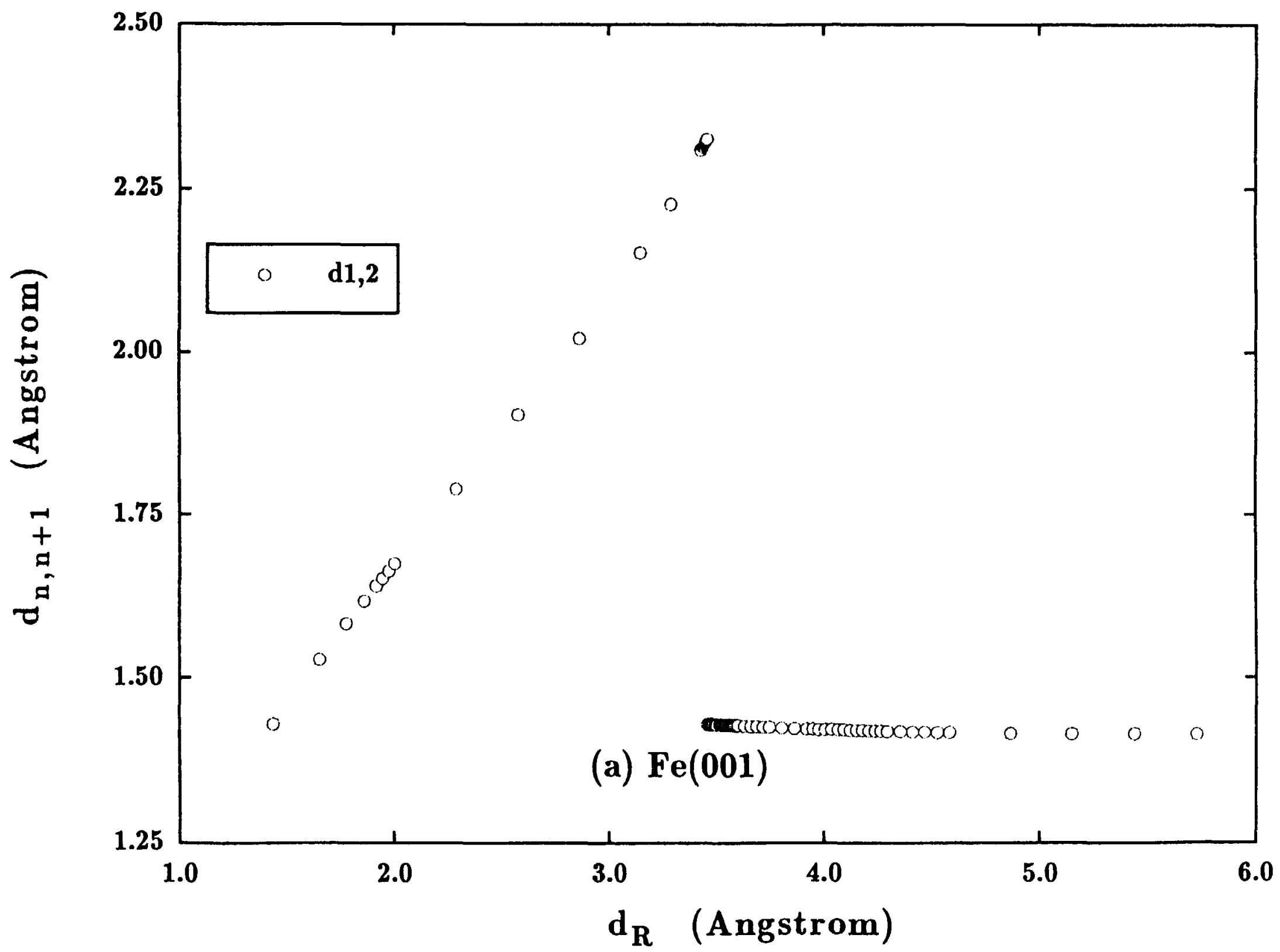




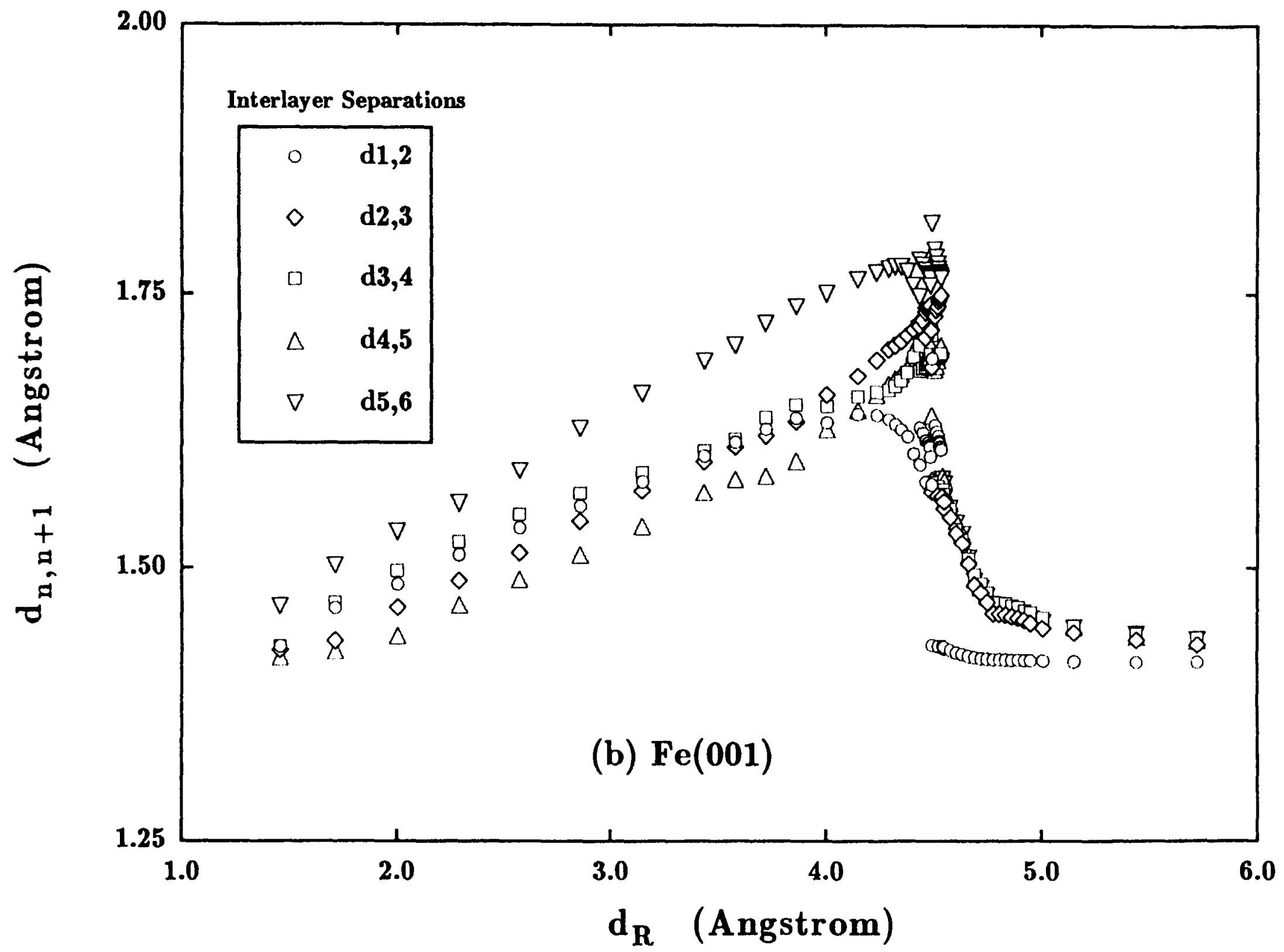




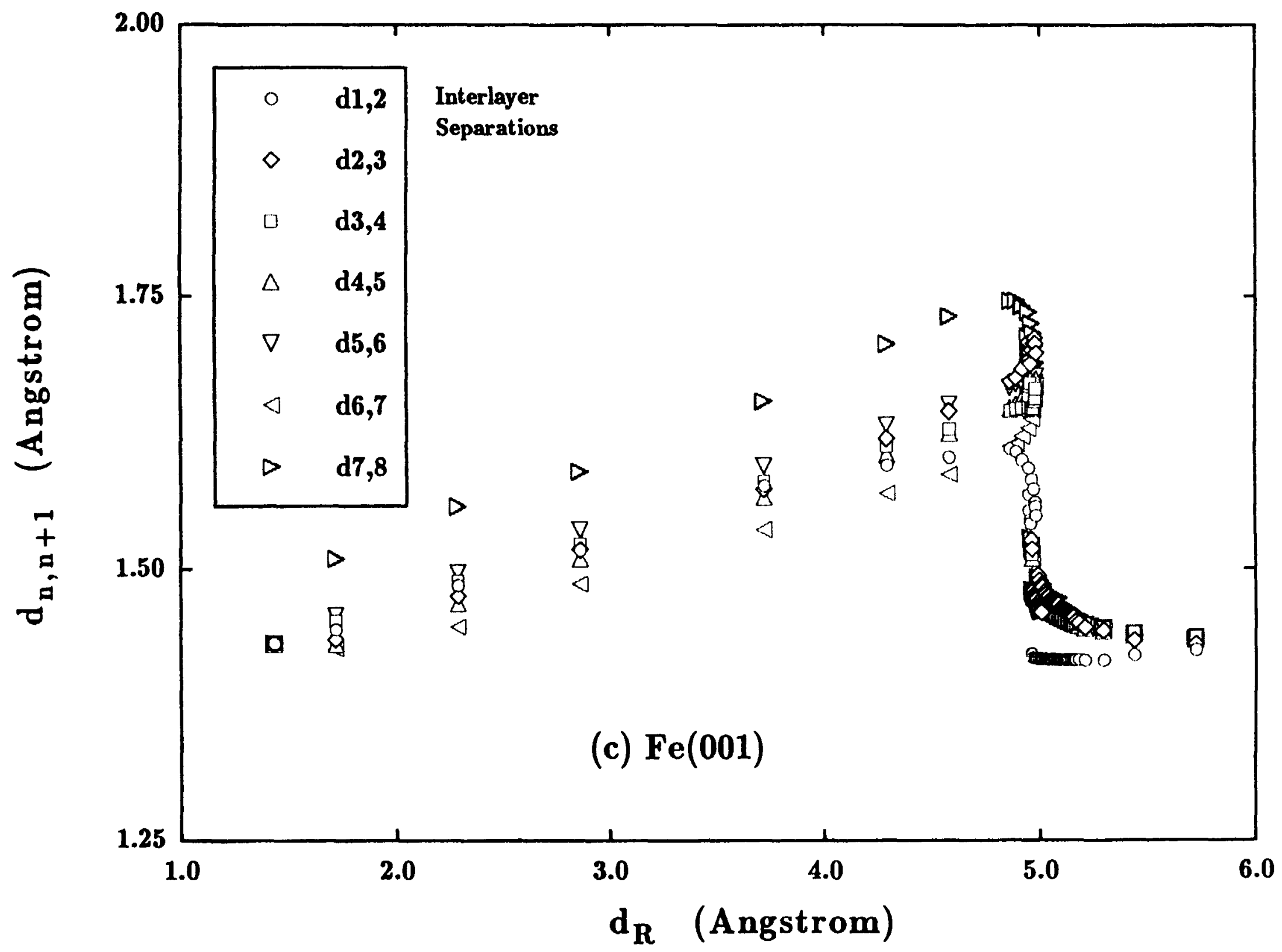




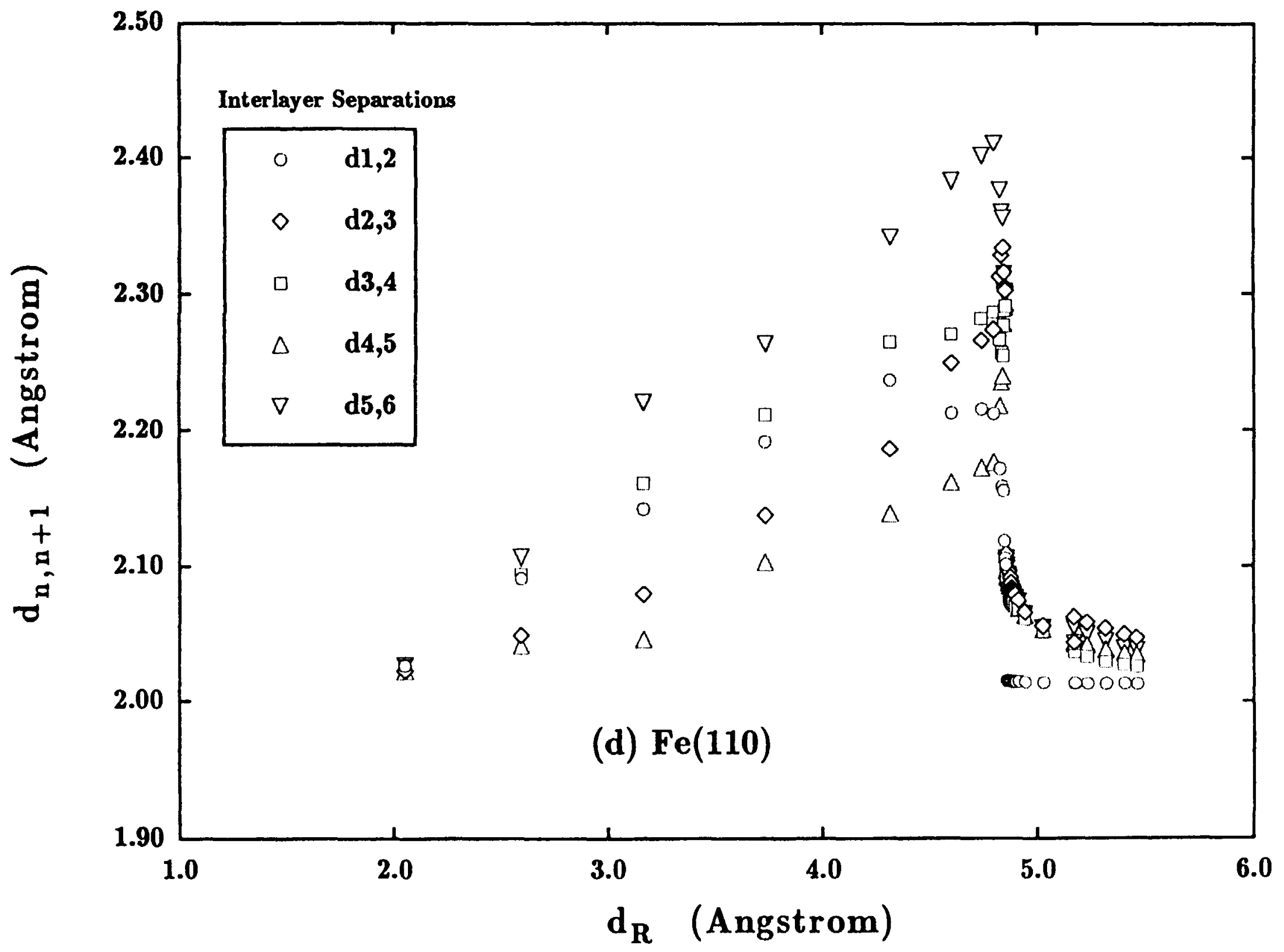




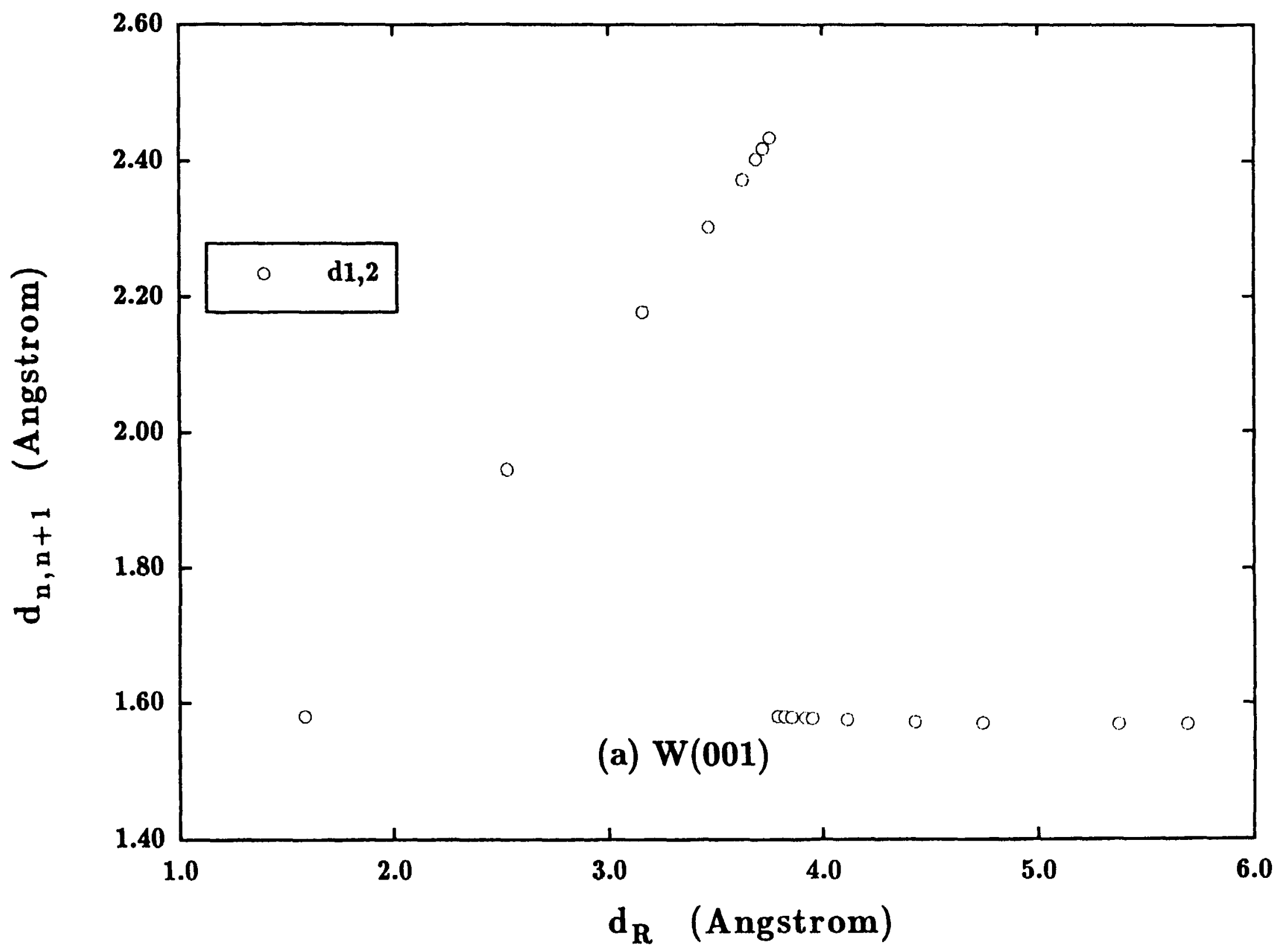




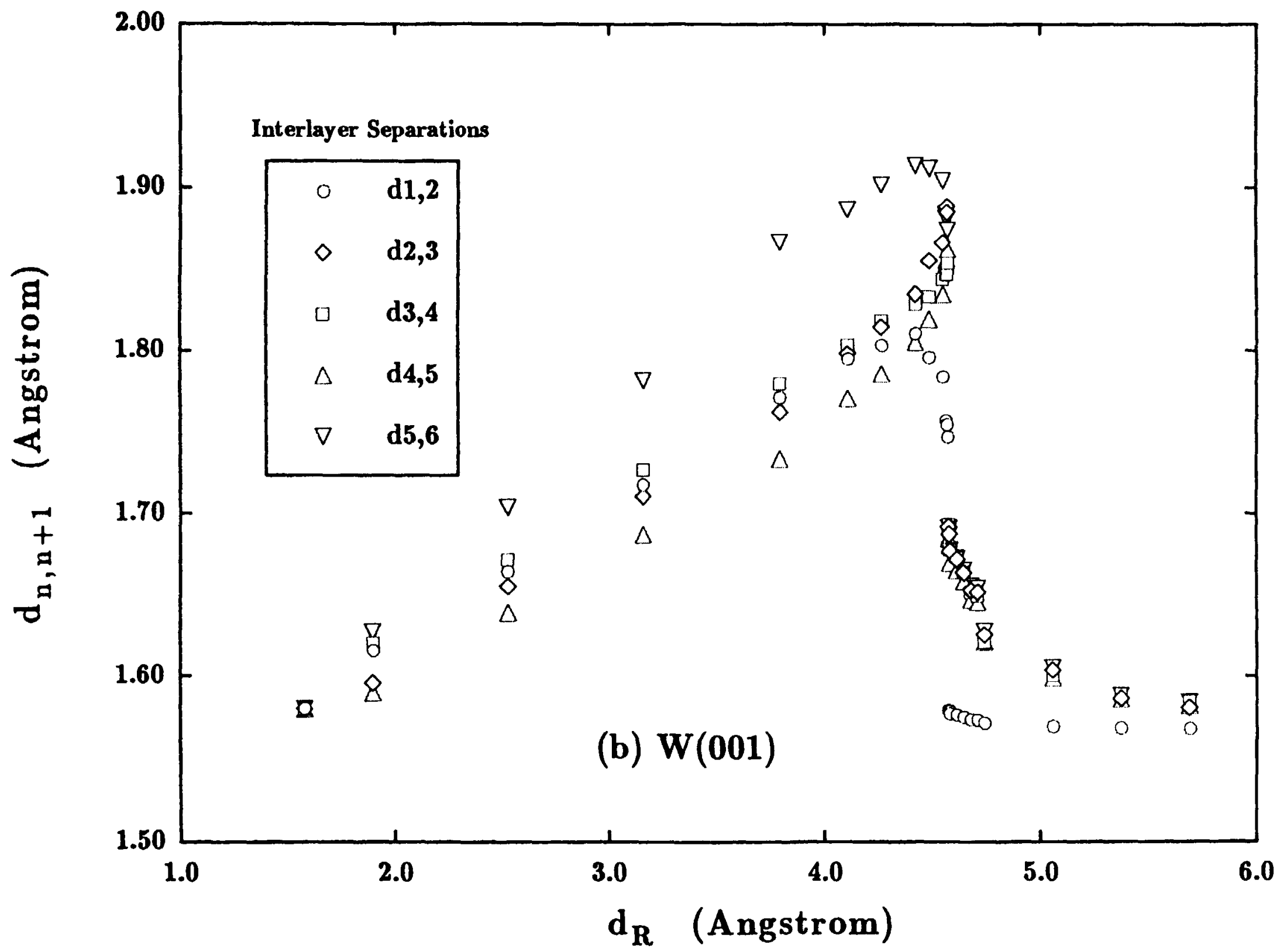




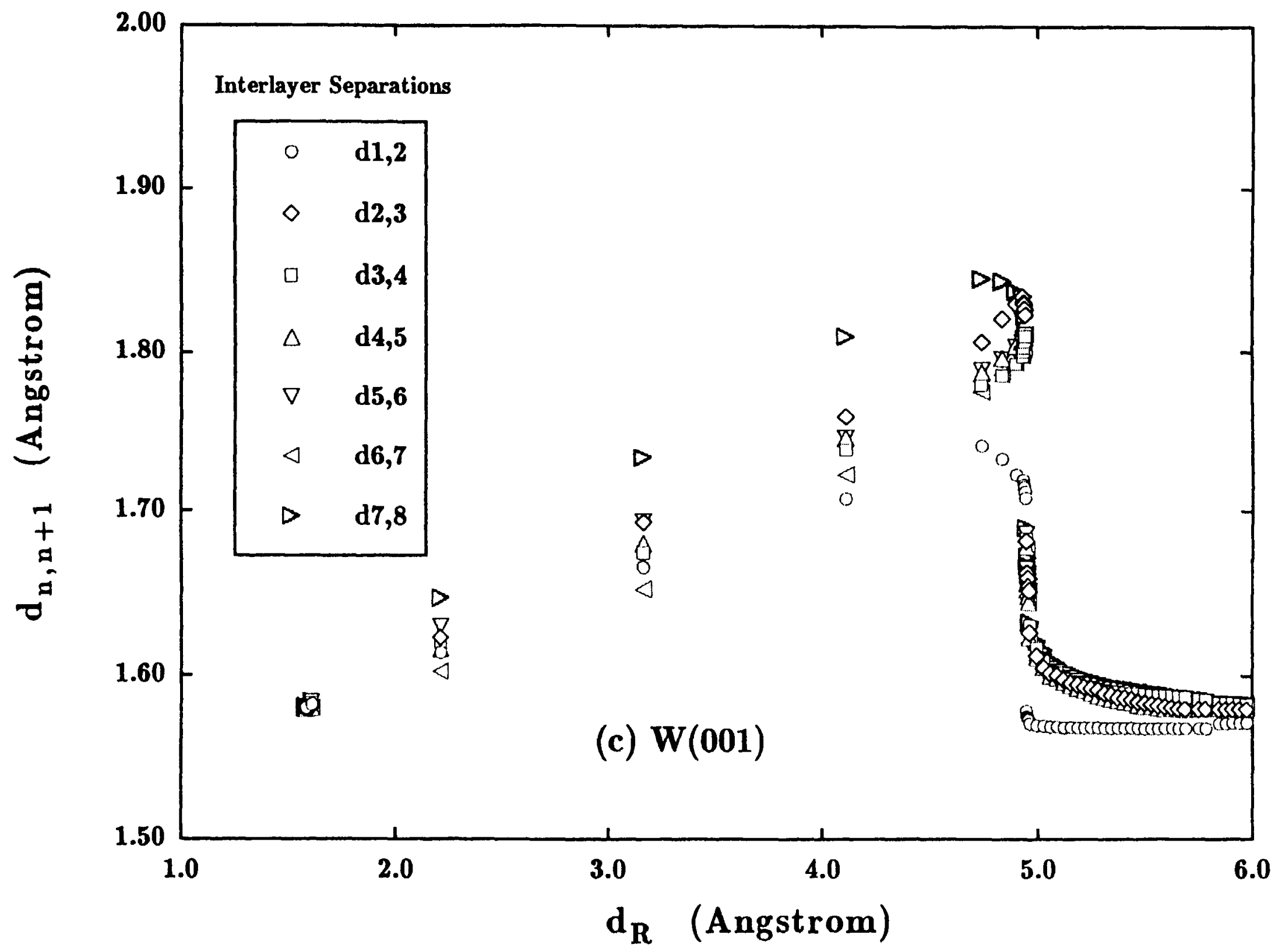




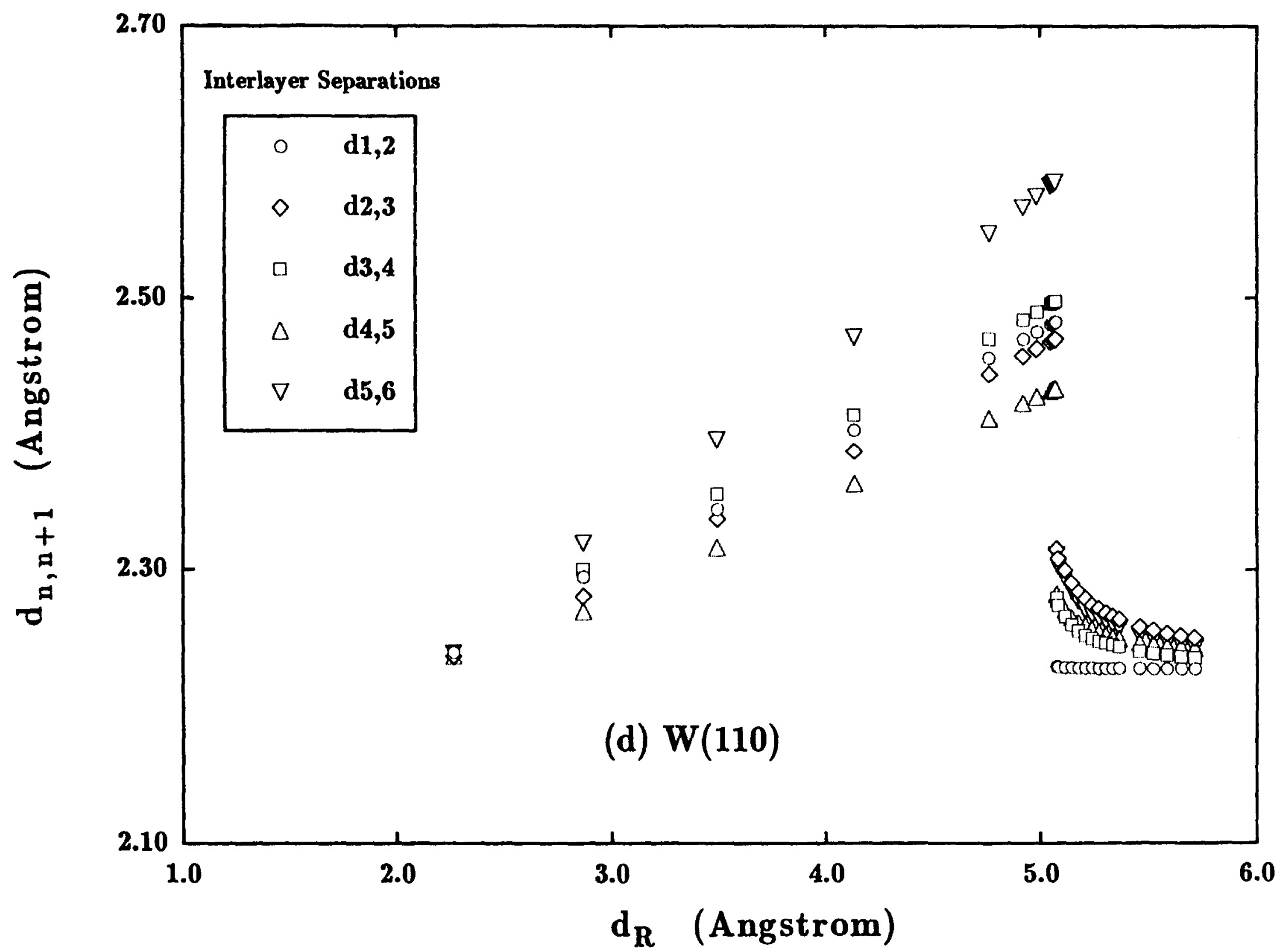




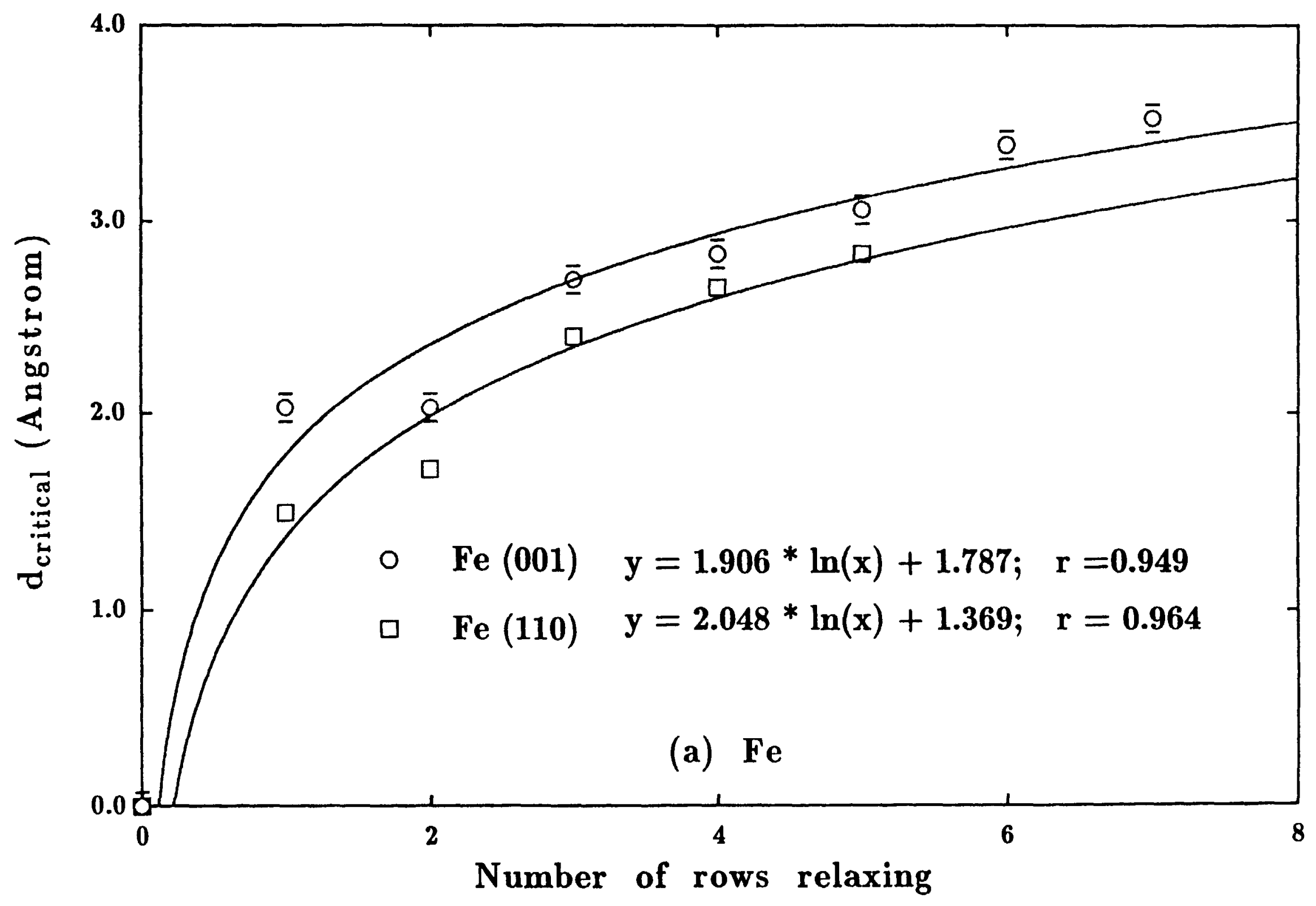




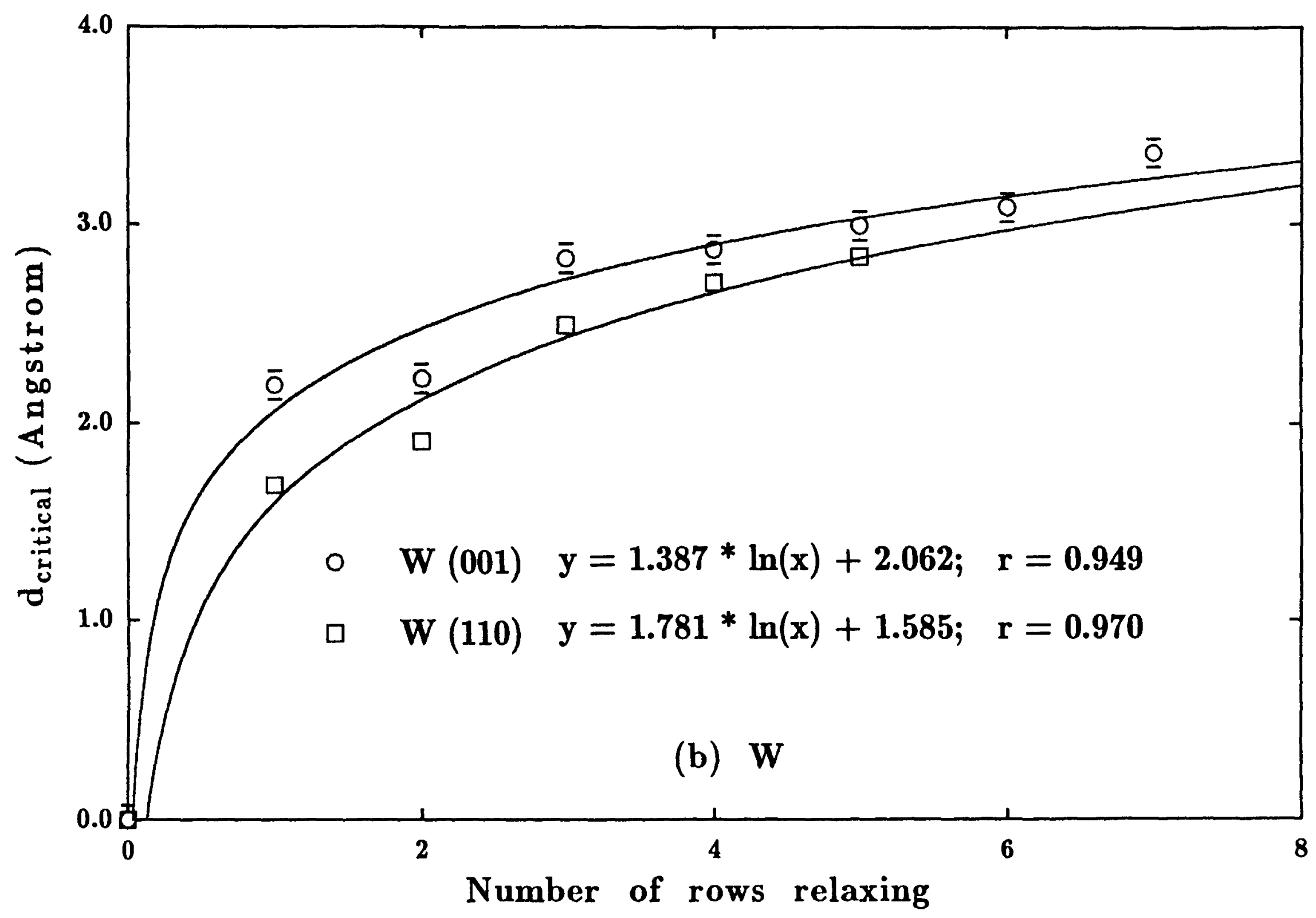




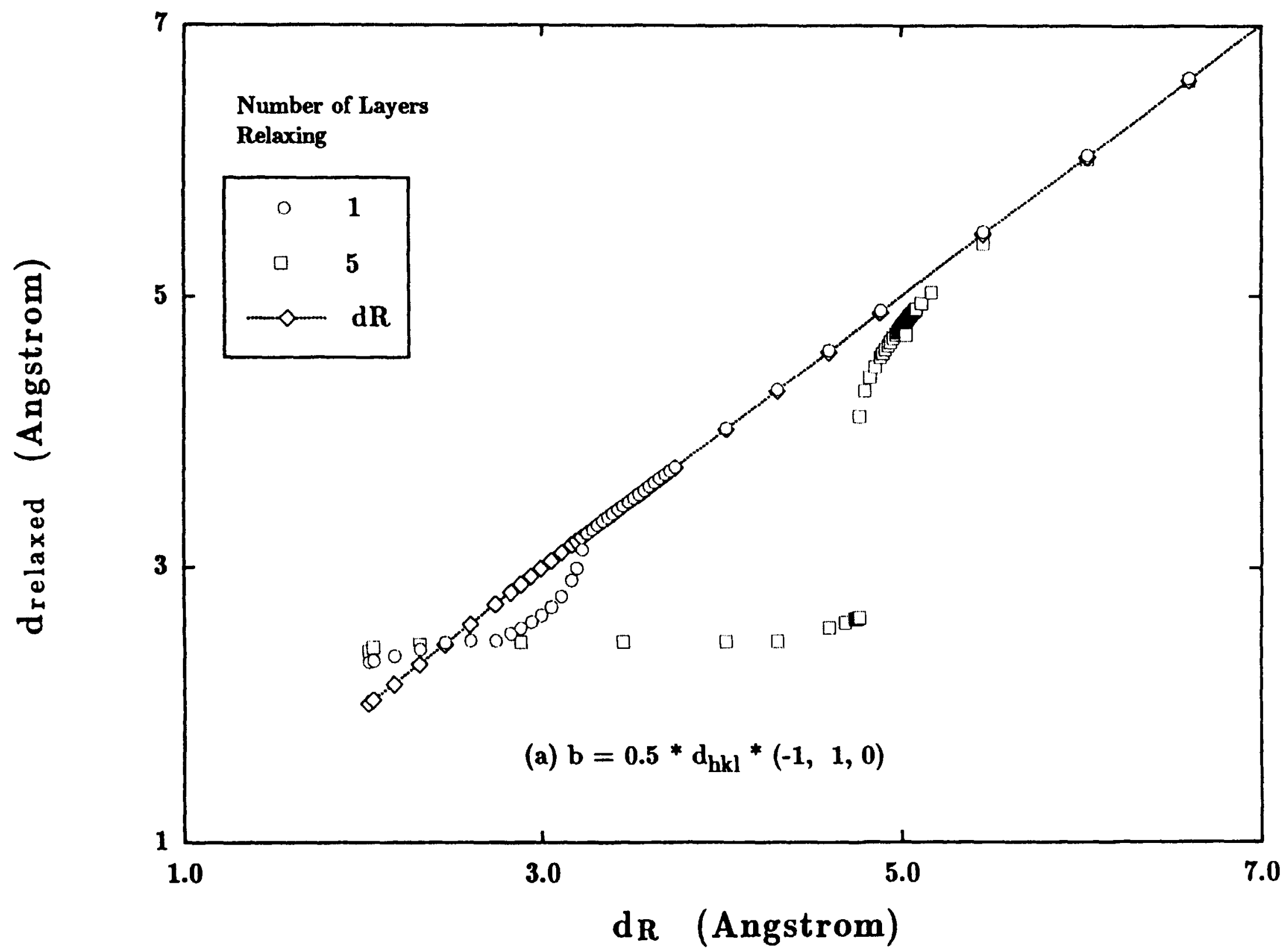




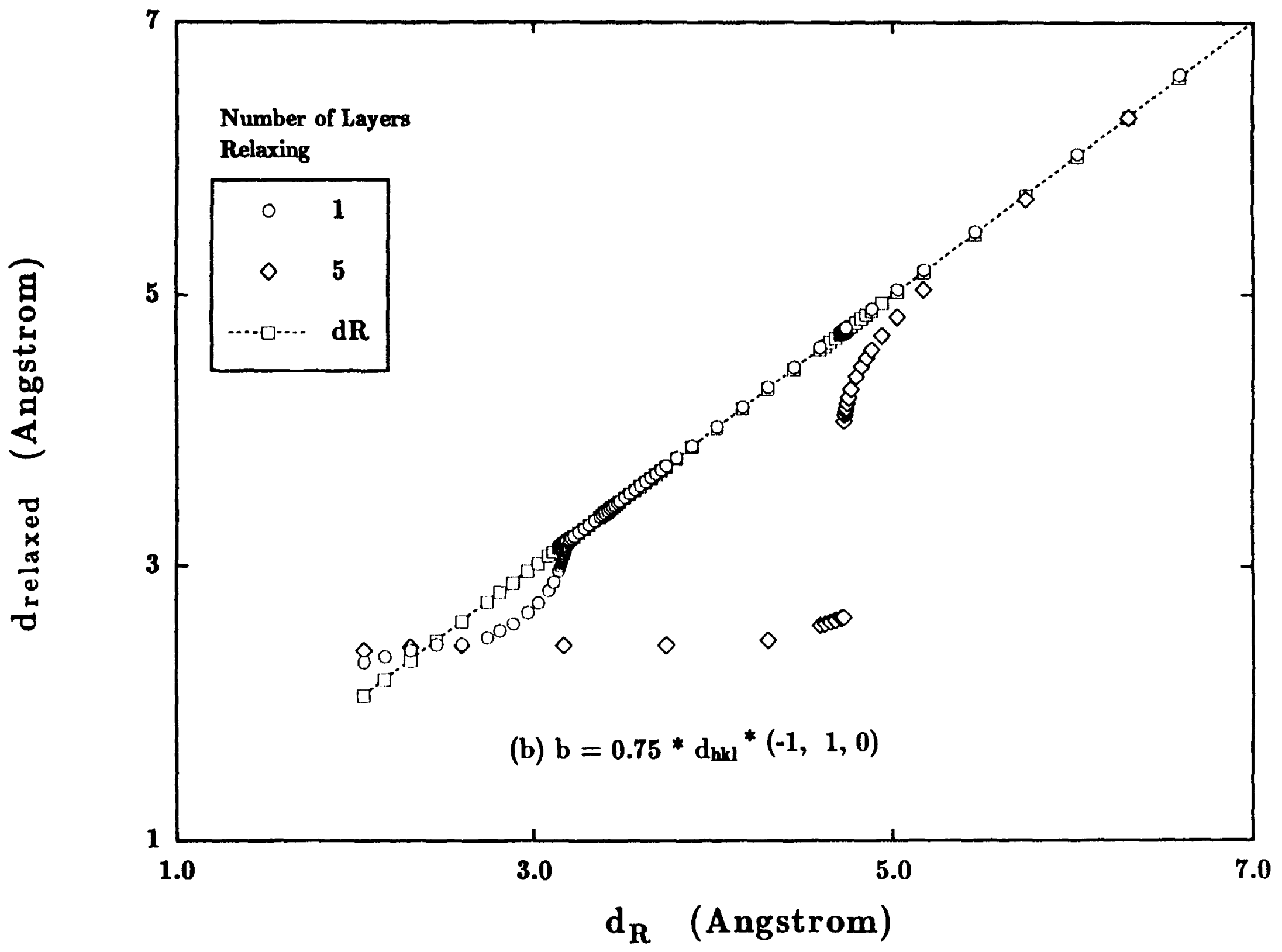




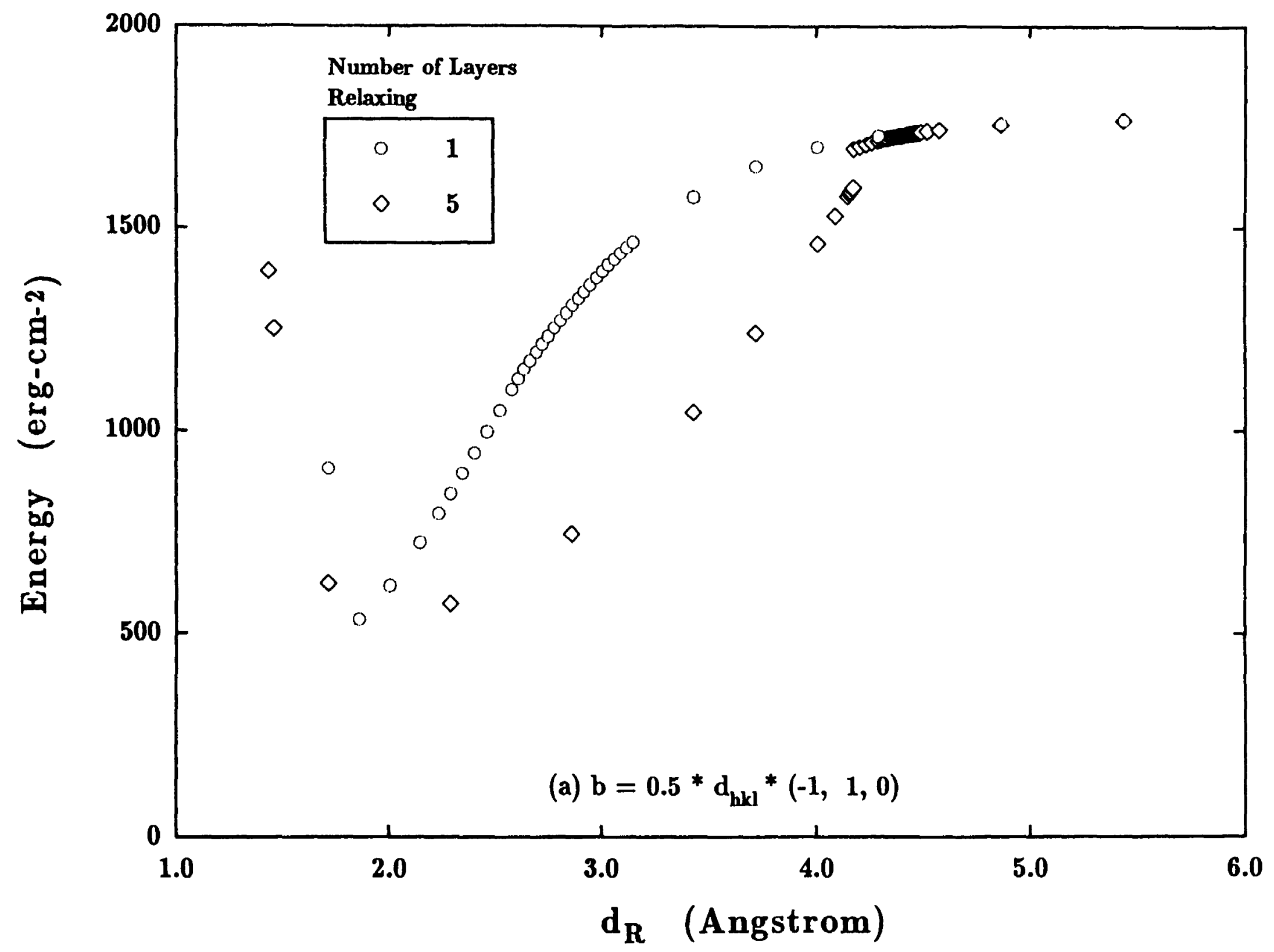




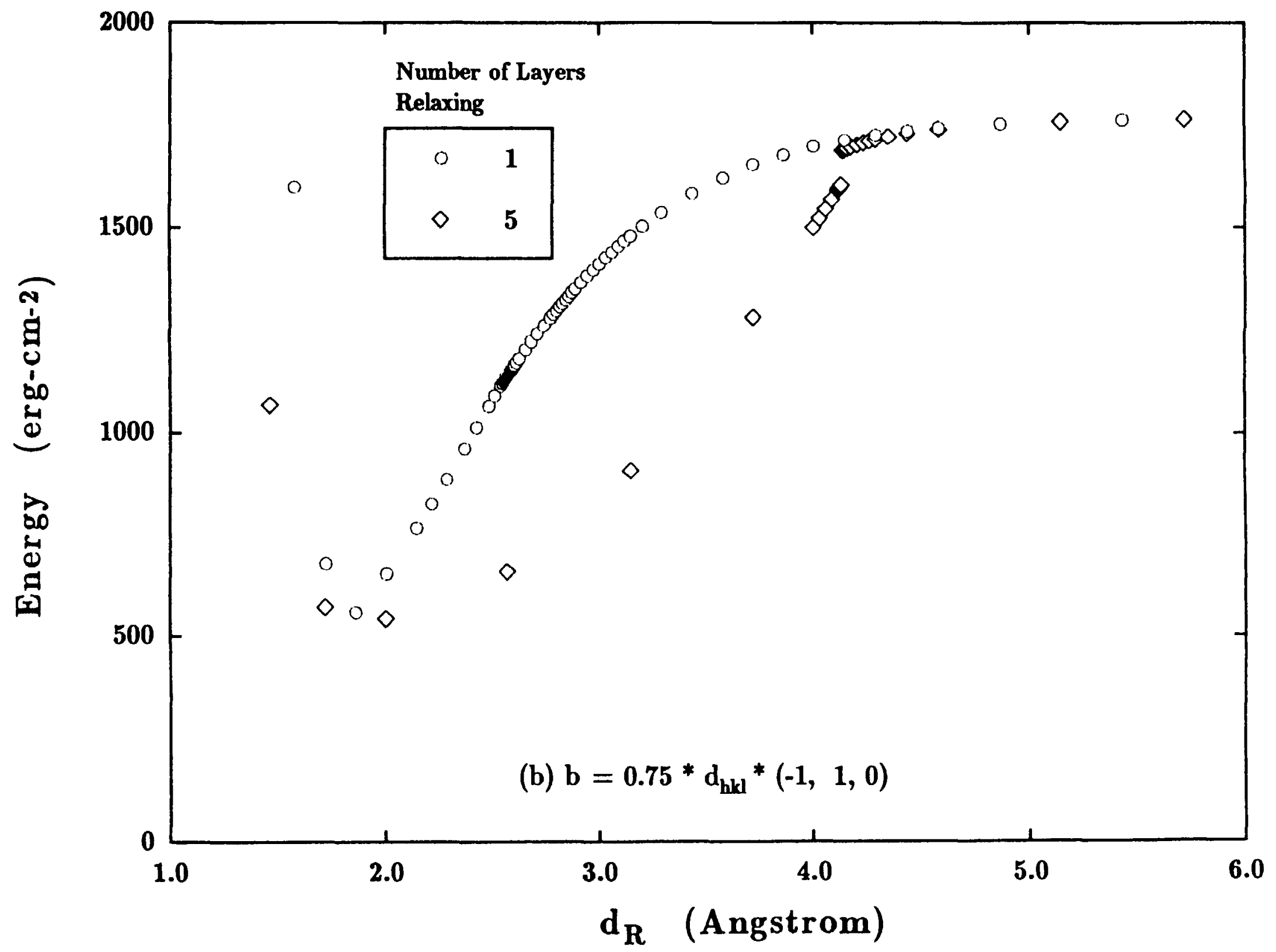




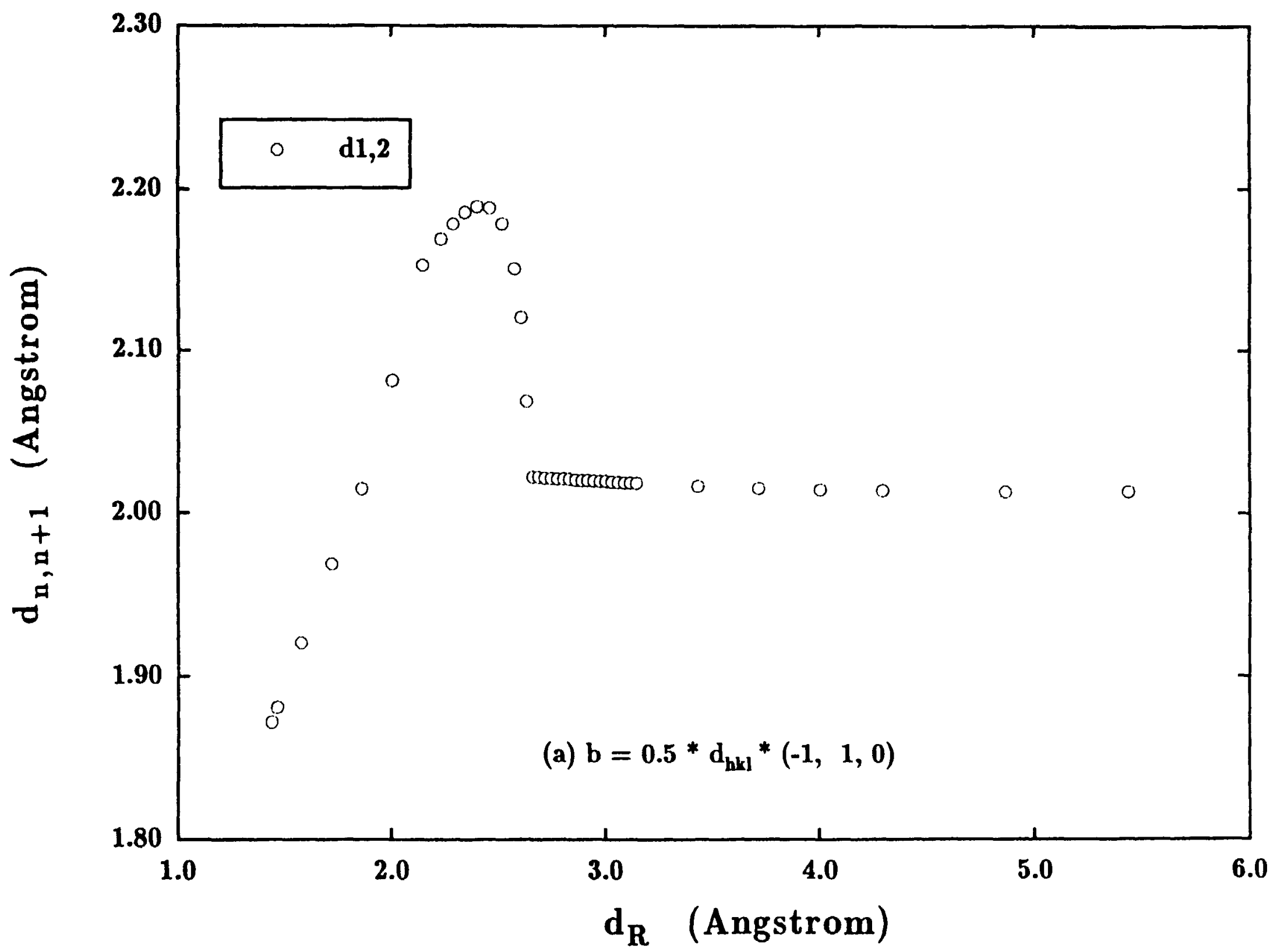




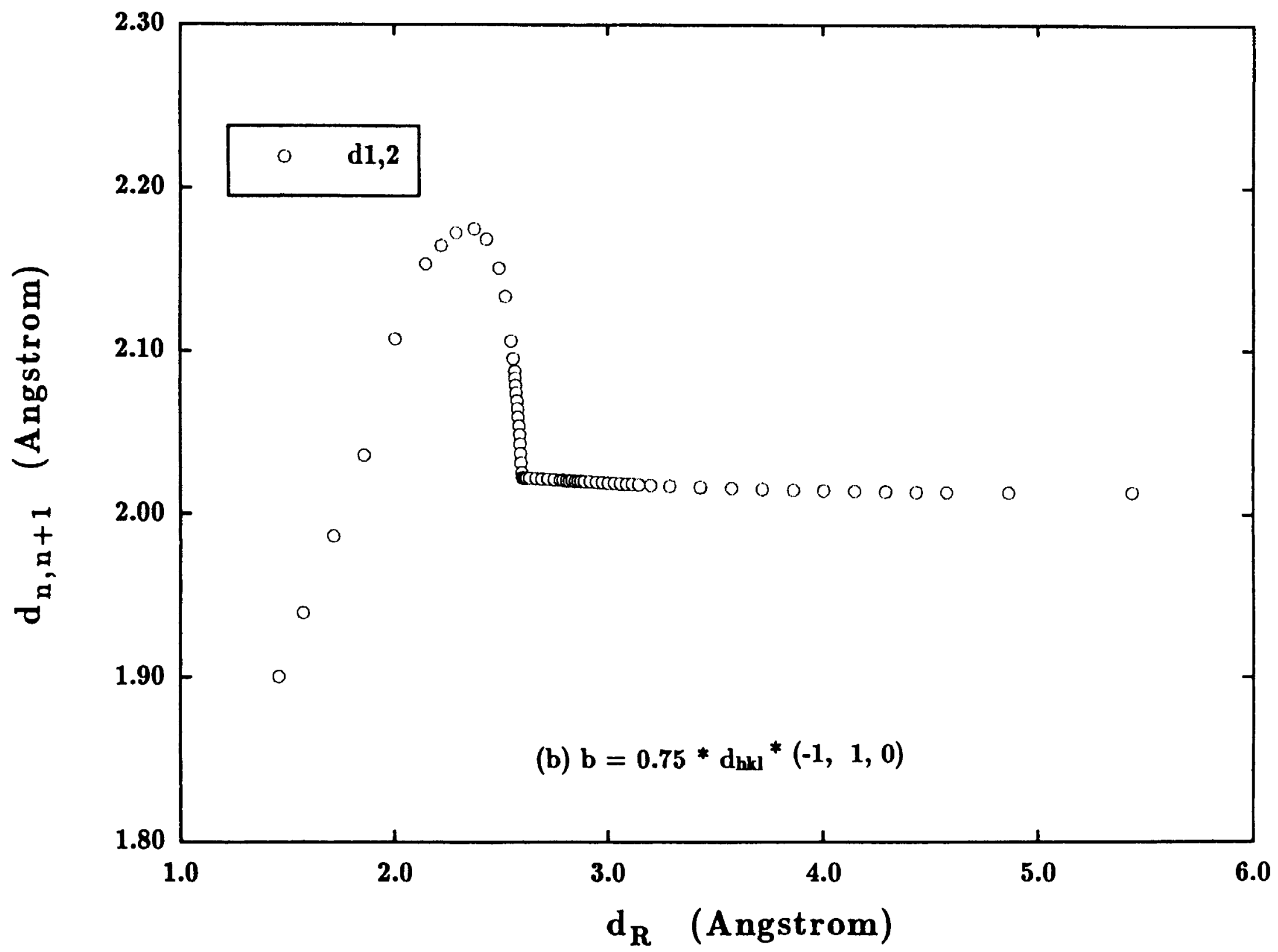




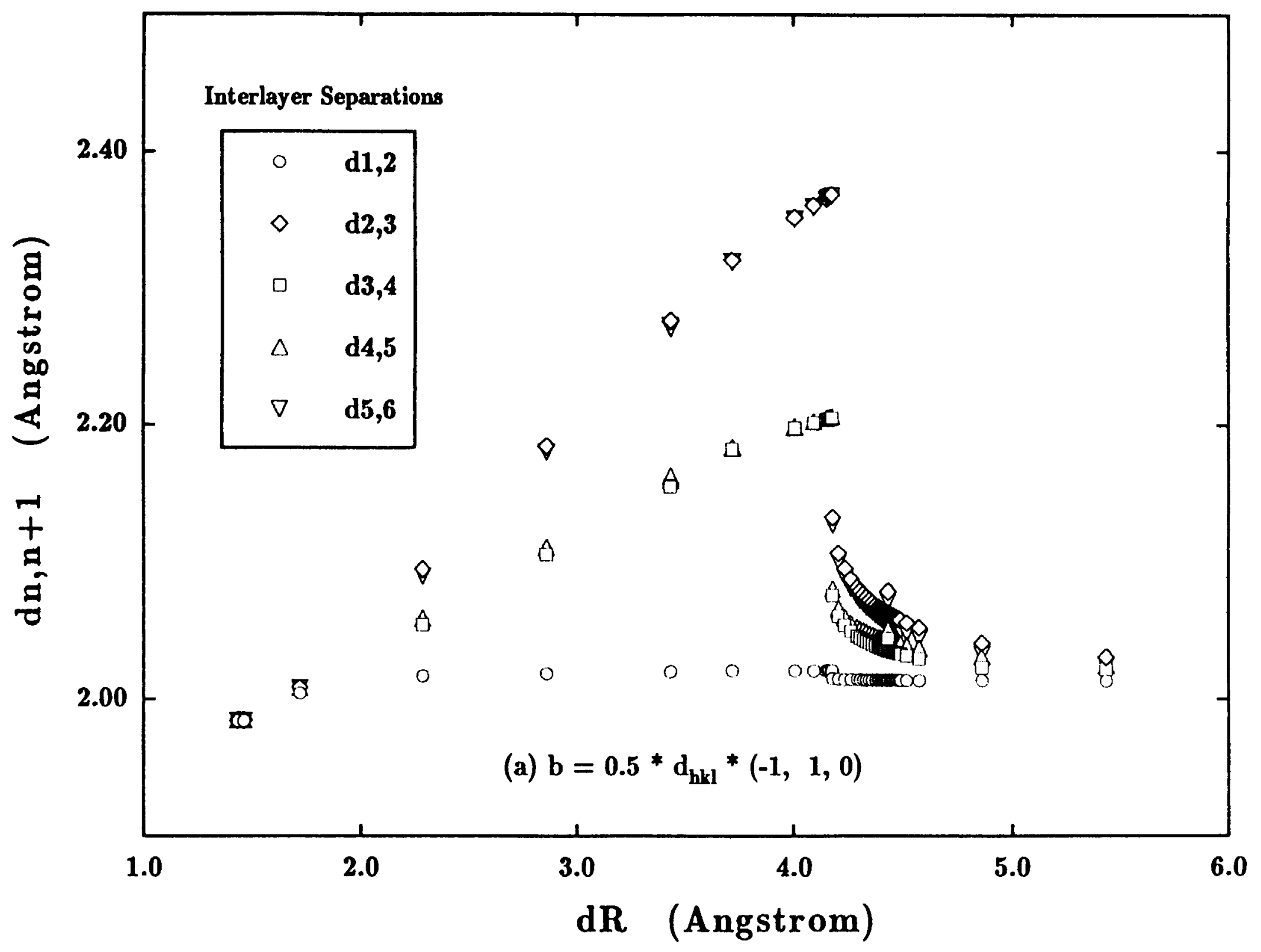




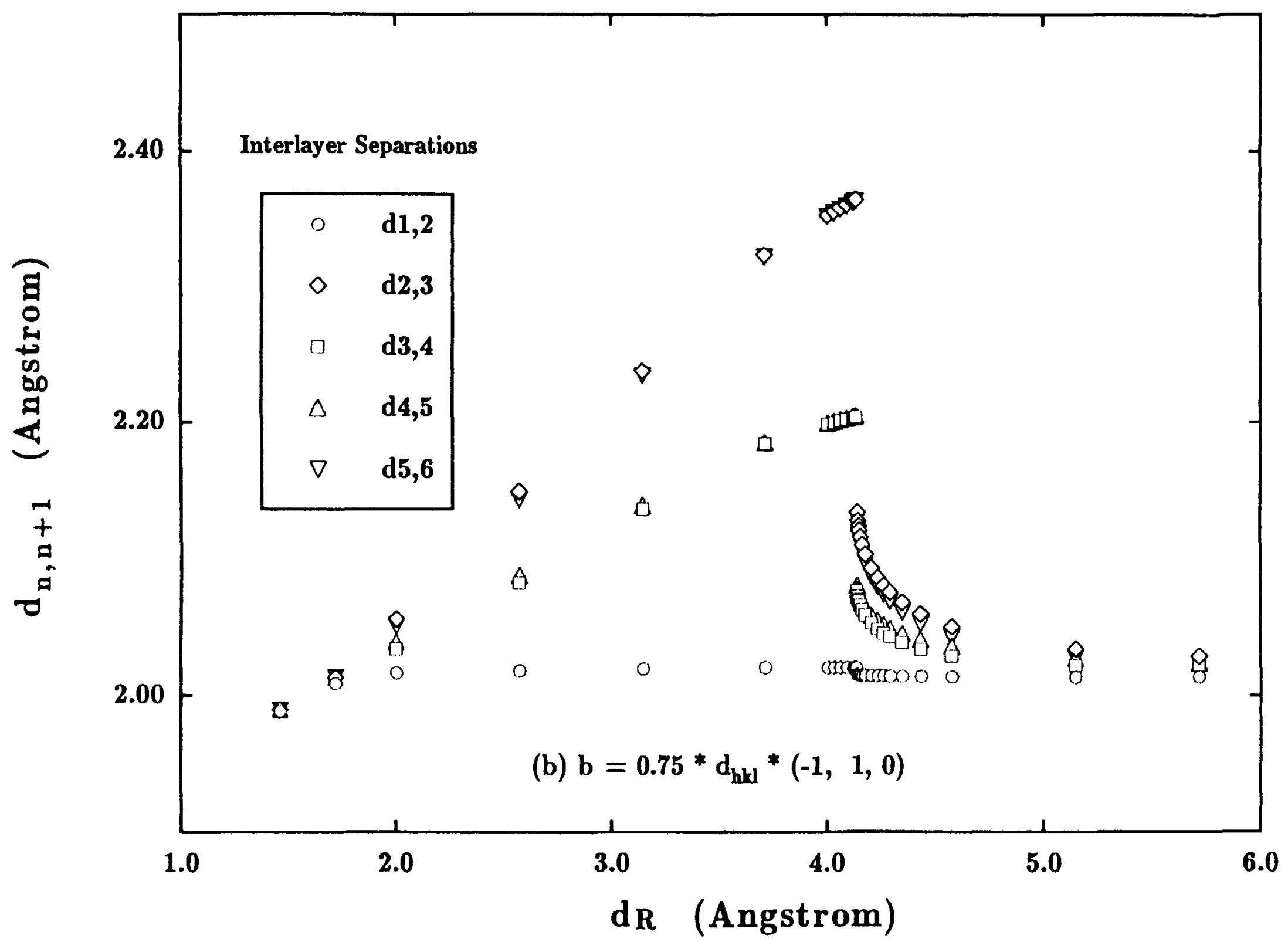

